TRANSACTIONS OF THE

AMERICAN MATHEMATICAL SOCIETY

Volume 359, Number 12, December 2007, Pages 5669-5696

S 0002-9947(07)04082-2

Article electronically published on June 25, 2007

\title{
GREEN'S FORMULAS FOR CONE DIFFERENTIAL OPERATORS
}

\author{
INGO WITT
}

\begin{abstract}
Green's formulas for elliptic cone differential operators are established. This is achieved by an accurate description of the maximal domain of an elliptic cone differential operator and its formal adjoint; thereby utilizing the concept of a discrete asymptotic type. From this description, the singular coefficients replacing the boundary traces in classical Green's formulas are deduced.
\end{abstract}

\section{INTRODUCTION}

1.1. The main result. Let $X$ be a compact $C^{\infty}$-manifold with non-empty boundary, $\partial X$. On the interior $X^{\circ}:=X \backslash \partial X$, we consider differential operators $A$ which on $U \backslash \partial X$ for some collar neighborhood $U \cong[0,1) \times Y$ of $\partial X$, with coordinates $(t, y)$ and $Y$ being diffeomorphic to $\partial X$, take the form

$$
A=t^{-\mu} \sum_{j=0}^{\mu} a_{j}\left(t, y, D_{y}\right)\left(-t \partial_{t}\right)^{j},
$$

where $a_{j} \in C^{\infty}\left([0,1)\right.$; $\left.\operatorname{Diff}^{\mu-j}(Y)\right)$ for $0 \leq j \leq \mu$. Such differential operators $A$ are called cone-degenerate, or of Fuchs type, and this is written as $A \in \operatorname{Diff}_{\text {cone }}^{\mu}(X)$. They arise, e.g., when polar coordinates are introduced near a conical point.

Throughout, we shall fix some reference weight $\delta \in \mathbb{R}$. This means that we will be working in the weighted $L^{2}$-space $\mathbb{H}^{0, \delta}(X)$ as the basic function space; see (1.12) and also Appendix B. Let $A^{*} \in \operatorname{Diff}_{\text {cone }}^{\mu}(X)$ be the formal adjoint to $A$, i.e,

$$
(A u, v)=\left(u, A^{*} v\right), \quad u, v \in C_{\mathrm{comp}}^{\infty}\left(X^{\circ}\right),
$$

where $\left(\right.$, ) denotes the scalar product in $\mathbb{H}^{0, \delta}(X)$. Then it is customary to consider the maximal and minimal domains of $A$,

$$
D\left(A_{\max }\right):=\left\{u \in \mathbb{H}^{0, \delta}(X) \mid A u \in \mathbb{H}^{0, \delta}(X)\right\},
$$

and $D\left(A_{\min }\right)$ is the closure of $C_{\text {comp }}^{\infty}\left(X^{\circ}\right)$ in $D\left(A_{\max }\right)$ with respect to the graph norm. Similarly for $D\left(A_{\max }^{*}\right), D\left(A_{\min }^{*}\right)$.

Our basic object of study is the boundary sesquilinear form

$$
[u, v]_{A}:=(A u, v)-\left(u, A^{*} v\right), \quad u \in D\left(A_{\max }\right), v \in D\left(A_{\max }^{*}\right) .
$$

Received by the editors October 26, 2003 and, in revised form, April 20, 2005.

2000 Mathematics Subject Classification. Primary 35J70; Secondary 34B05, 41A58.

Key words and phrases. Cone differential operators, discrete asymptotic types, function spaces with asymptotics, complete conormal symbols, Keldysh's formula, Green's formula.

(C)2007 American Mathematical Society Reverts to public domain 28 years from publication 
By virtue of (1.2), $[u, v]_{A}=0$ if $u \in D\left(A_{\min }\right)$ or if $v \in D\left(A_{\min }^{*}\right)$. Therefore, the boundary sesquilinear form $[,]_{A}$ descends to a sesquilinear form

$$
[,]_{A}: D\left(A_{\max }\right) / D\left(A_{\min }\right) \times D\left(A_{\max }^{*}\right) / D\left(A_{\min }^{*}\right) \rightarrow \mathbb{C},
$$

denoted in the same manner.

Our basic task consists in computing (1.4). The result will be called a Green's formula in analogy to the classical situation arising in mathematical analysis. Assuming ellipticity for $A$, see Definition 3.1, what we will actually do is to compute the value of $[,]_{A}$ on distinguished linear bases of $D\left(A_{\max }\right) / D\left(A_{\min }\right)$ and $D\left(A_{\max }^{*}\right) / D\left(A_{\min }^{*}\right)$, respectively.

We start off with the following observation: if $A$ is elliptic, then any solution $u=u(x)$ to the equation $A u=f(x)$ on $X^{\circ}$ possesses an asymptotic expansion

$$
u(x) \sim \sum_{p} \sum_{k+l=m_{p}-1} \frac{(-1)^{k}}{k !} t^{-p} \log ^{k} t \phi_{l}^{(p)}(y) \quad \text { as } t \rightarrow+0,
$$

where $p \in \mathbb{C}, m_{p} \geq 0$ is an integer depending on $p$, the set $\left\{p \in \mathbb{C} \mid m_{p} \geq 1\right\}$ is discrete, $\operatorname{Re} p \rightarrow-\infty$ as $|p| \rightarrow \infty$ on this set, and $\phi_{l}^{(p)} \in C^{\infty}(Y)$ for all $p, l$, provided that the right-hand side $f(x)$ possesses a similar expansion. Introduce the linear operator $T$ acting on the space of all formal asymptotic expansions of the form (1.5) by

$$
\begin{aligned}
\sum_{p} \sum_{k+l=\underline{m_{p}-1}} \frac{(-1)^{k}}{k !} t^{-p} \log ^{k} t \phi_{l}^{(p)} & (y) \\
& \mapsto \sum_{p} \sum_{k+l=\underline{m_{p}-2}} \frac{(-1)^{k}}{k !} t^{-p} \log ^{k} t \phi_{l}^{(p)}(y) .
\end{aligned}
$$

As will be seen,

- the quotient $D\left(A_{\max }\right) / D\left(A_{\min }\right)$ is finite-dimensional,

- it can naturally be identified with a space consisting of finite sums of the form (1.5), where $\operatorname{dim} X / 2-\delta-\mu<\operatorname{Re} p<\operatorname{dim} X / 2-\delta$,

- when doing so, this quotient is left invariant under the action of $T$.

In particular, $T$ as acting on $D\left(A_{\max }\right) / D\left(A_{\min }\right)$ is nilpotent. Similarly for the quotient $D\left(A_{\max }^{*}\right) / D\left(A_{\min }^{*}\right)$.

Then our main result states:

Theorem 1.1. Let $A \in \operatorname{Diff}_{\text {cone }}^{\mu}(X)$ be elliptic. Then, to each Jordan basis

$$
\Phi_{1}, T \Phi_{1}, \ldots, T^{m_{1}-1} \Phi_{1}, \ldots, \Phi_{e}, T \Phi_{e}, \ldots, T^{m_{e}-1} \Phi_{e}
$$

of $D\left(A_{\max }\right) / D\left(A_{\min }\right)$, there exists a unique Jordan basis

$$
\Psi_{1}, T \Psi_{1}, \ldots, T^{m_{1}-1} \Psi_{1}, \ldots, \Psi_{e}, T \Psi_{e}, \ldots, T^{m_{e}-1} \Psi_{e}
$$

of $D\left(A_{\max }^{*}\right) / D\left(A_{\min }^{*}\right)$ such that, for all $i, j, r, s$,

$$
\left[T^{r} \Phi_{i}, T^{s} \Psi_{j}\right]_{A}= \begin{cases}(-1)^{s+1} & \text { if } i=j, r+s=m_{i}-1 \\ 0 & \text { otherwise }\end{cases}
$$


Corollary 1.2. (a) Both spaces $D\left(A_{\max }\right) / D\left(A_{\min }\right)$ and $D\left(A_{\max }^{*}\right) / D\left(A_{\min }^{*}\right)$ have the same Jordan structure (with respect to $T$ ).

(b) The sesquilinear form $[,]_{A}$ in (1.4) is non-degenerate.

(c) The operator $T$ is skew-adjoint with respect to $[,]_{A}$, i.e.,

$$
[T \Phi, \Psi]_{A}+[\Phi, T \Psi]_{A}=0
$$

for all $\Phi \in D\left(A_{\max }\right) / D\left(A_{\min }\right), \Psi \in D\left(A_{\max }^{*}\right) / D\left(A_{\min }^{*}\right)$.

Remark 1.3. (a) The conjugate Jordan basis $\Psi_{1}, T \Psi_{1}, \ldots, T^{m_{1}-1} \Psi_{1}, \ldots, \Psi_{e}, T \Psi_{e}$, $\ldots, T^{m_{e}-1} \Psi_{e}$ in (1.8) can be found, at least in principle, once one controls the first $\mu$ conormal symbols $\sigma_{c}^{\mu-j}(A)(z)$ for $j=0,1, \ldots, \mu-1$ of $A$; see (3.1). More precisely, let $\left\{\mathfrak{t}^{-\mu-k}(z) ; k \in \mathbb{N}_{0}\right\}$ be the inverse to the complete conormal symbol $\left\{\sigma_{c}^{\mu-j}(A)(z) ; j \in \mathbb{N}_{0}\right\}$ of $A$ under the Mellin translation product; see (3.5). In particular, $\mathfrak{t}^{-\mu-k}(z)$ for $k=0,1,2, \ldots$ is a meromorphic function on $\mathbb{C}$ taking values in the space $L_{\mathrm{cl}}^{-\mu}(Y)$ of classical pseudodifferential operators of order $-\mu$ on $Y$. Then the Jordan basis in (1.8) can be computed from the Jordan basis in (1.7) and from the knowledge of the principal parts of the Laurent expansions of $\mathfrak{t}^{-\mu-k}(z+\mu)$ around the poles in the strip $\operatorname{dim} X / 2-\delta-\mu+k<\operatorname{Re} z<\operatorname{dim} X / 2-\delta$ for $k=0,1, \ldots, \mu-1$. For details see Theorem 4.1 .

(b) Theorem 1.1 remains true for cone differential operators $A$ acting between sections of vector bundles over $X$, under the same ellipticity assumption. For the sake of simplicity, we confine ourselves to the scalar case.

1.2. Description of the content. In Section 2, we discuss discrete asymptotic types for conormal asymptotics of the form (1.5). The central notions are properness of an asymptotic type and complete characteristic bases for proper asymptotic types. In Section 3, we study complete Mellin symbols that form an algebra unter the Mellin translation product. Here, the main result due to LIU-WiTT 12 states that the type for the asymptotics annihilated by an elliptic, holomorphic complete Mellin symbol is proper; thus linking to cone differential operators, see Theorem 3.10. Then in Theorem 4.1, in Section 4 we establish a formula for the principal parts of the Laurent expansions around the poles of the inverses to holomorphic complete Mellin symbols under the Mellin translation product. This formula involves a complete characteristic basis and its conjugate complete characteristic basis, similar to the situation arising in Theorem 1.1. In technical terms, Theorem 4.1 is one of the two main results of this paper from which Theorem 1.1 is easily deduced. The other one is Theorem 5.1 in Section 5, where certain "bi-orthogonality" relations between the two complete characteristic bases of Theorem 4.1 are established. Theorem 1.1 is proved in Section 6. We start with a formula for the boundary sesquilinear form $[,]_{A}$ taken from GIL-MendozA [4; see Theorem 6.1. The proof of Theorem 1.1 then consists in evaluating this formula, where the latter basically means to "take the residue" of the formula from Theorem 5.1 .

In Section 7, we discuss two examples showing how one can proceed from "Green's formula" (1.9) to genuine Green's formulas in concrete situations. With the exception of formula (B.4), the two appendices are not necessary for the main text, but improve understanding. In Appendix $\mathrm{A}$, we are concerned with local asymptotic types, i.e., asymptotic types at some fixed singular exponent $p$ from (1.5). Already here, all the ingredients from the main text of the paper occur in embryonic form. For instance, the forerunner of Theorem 5.1 is a famous formula due to KELDYSH [7; see Remark 5.2 (b). An analogue of the boundary sesquilinear form $[,]_{A}$ is also 
provided; see (A.3) and Proposition A.4 In Appendix B. we describe $D\left(A_{\max }\right)$, $D\left(A_{\min }\right)$ as function spaces with asymptotics. Among others, this gives a concise way of identifying the quotient $D\left(A_{\max }\right) / D\left(A_{\min }\right)$.

Let us mention some related work: Green's formulas have been under investigation for a long time; see, e.g., CodDington-Levinson [1] for O.D.E. and Lions-Magenes [11] for P.D.E. For singular situations, see, e.g., NAZARovPlamenevskiJ 14. Our approach to cone-degenerate differential operators is built upon work of ScHUlze [16, 17]. For instance, the fact that the quotient $D\left(A_{\max }\right) / D\left(A_{\min }\right)$ is finite-dimensional and can be identified with a space of formal asymptotic expansions of finite length is an easy consequence; see also LESCH 10, Section 1.3]. Recently, GiL-Mendoza [4 obtained results similar to ours. Without reaching the final formula (1.9), they studied much of the structure of the boundary sesquilinear form (1.4). In case $A$ is symmetric, they applied their results to describe the self-adjoint extensions of $A$. Keldysh's formula was thoroughly discussed in KozLOV-Maz'ya [9, Appendix A].

1.3. Notation. Notation introduced here will be used without further comment.

- Scalar products on $L^{2}(Y)$ are given by

$$
(\phi, \psi):=\int_{Y} \phi(y) \overline{\psi(y)} d \mu(y), \quad\langle\phi, \psi\rangle:=\int_{Y} \phi(y) \psi(y) d \mu(y),
$$

where $d \mu$ is a fixed positive $C^{\infty}$-density $d \mu$ on $Y$. For an operator $B$ on $C^{\infty}(Y)$, its formal adjoint $B^{*}$ is defined with respect to the scalar product $($,$) , while the$ transpose $B^{t}$ is defined with respect to $\langle$,$\rangle . In particular,$

$$
\overline{B^{*} \phi}=B^{t} \bar{\phi}, \quad \phi \in C^{\infty}(Y) .
$$

For $u, v \in \mathbb{H}^{0, \delta}(X)$, where one of them is supported in the collar neighborhood $U$ of $\partial X$,

$$
(u, v):=\iint_{(0,1) \times Y} t^{\operatorname{dim} X-2 \delta-1} u(t, y) \overline{v(t, y)} d t d \mu(y) .
$$

There should be no ambiguity of usage for the same symbol $($,$) in the two different$ situations (1.11), (1.12).

- Let $J$ be a finite-dimensional linear space and let $T$ be a nilpotent operator acting on $J$. Then $\Phi_{1}, \ldots, \Phi_{e}$ is called a characteristic basis of $J$ (with respect to $T$, where the latter is often understood from the context) if

$$
\Phi_{1}, T \Phi_{1}, \ldots, T^{m_{1}-1} \Phi_{1}, \ldots, \Phi_{e}, T \Phi_{e}, \ldots, T^{m_{e}-1} \Phi_{e},
$$

for certain integers $m_{1}, \ldots, m_{e} \geq 1$, form a linear basis of $J$. The matrix of $T$ with respect to such a linear basis has Jordan form. Therefore, a characteristic basis $\Phi_{1}, \ldots, \Phi_{e}$ always exists, the integers $m_{1}, \ldots, m_{e}$ are uniquely determined (up to permutation) and equal the sizes of the Jordan blocks, and $e$ is the number of the Jordan blocks. The tuple $\left(m_{1}, \ldots, m_{e}\right)$ is called the characteristic of $J$ (or of the characteristic basis $\left.\Phi_{1}, \ldots, \Phi_{e}\right)$.

- Let $E$ be either the space $C^{\infty}(Y)$ (in Section 2) or a Banach space (in Appen$\operatorname{dix}$ A). Then $E^{\infty}:=\bigcup_{m \in \mathbb{N}} E^{m}$ denotes the space of finite sequences in $E$, where we identify $E^{m}$ as a linear subspace of $E^{m+1}$ via the mapping $\left(\phi_{0}, \ldots, \phi_{m-1}\right) \mapsto$ $\left(0, \phi_{0}, \ldots, \phi_{m-1}\right)$, i.e., by adding a leading zero. For $\Phi \in E^{\infty}$, let $m(\Phi)$ be the least integer $m$ so that $\Phi \in E^{m}$. The right shift operator $T$ sending $\left(\phi_{0}, \phi_{1}, \ldots, \phi_{m-1}\right)$ to $\left(\phi_{0}, \phi_{1}, \ldots, \phi_{m-2}\right)$ acts on $E^{\infty}$. In particular, $T^{m(\Phi)} \Phi=0$, while $T^{i} \Phi \neq 0$ for 
$0 \leq i \leq m(\Phi)-1$. (In case $E=C^{\infty}(Y)$, the operator $T$ is directly related to the operator $T$ in (1.6); see Remark 2.2.)

- For $E$ as above, $p \in \mathbb{C}$, let $\mathcal{M}_{p}(E)$ be the space of germs of $E$-valued meromorphic functions and let $\mathcal{A}_{p}(E)$ be the space of germs of $E$-valued holomorphic functions at $z=p$. We identify the quotient $\mathcal{M}_{p}(E) / \mathcal{A}_{p}(E)$ with the space $E^{\infty}$ via the mapping

$$
\frac{\phi_{0}}{(z-p)^{m}}+\frac{\phi_{1}}{(z-p)^{m-1}}+\cdots+\frac{\phi_{m-1}}{z-p} \mapsto\left(\phi_{0}, \phi_{1}, \ldots, \phi_{m-1}\right) .
$$

Then $T$ corresponds to multiplication by $z-p$. For $F \in \mathcal{M}_{p}(E)$, let $[F(z)]_{p}^{*}$ denote the principal part of $F(z)$ at $z=p$. For $\Phi=\left(\phi_{0}, \phi_{1}, \ldots, \phi_{m-1}\right) \in E^{\infty}$, we set

$$
\Phi[z-p]:=\frac{\phi_{0}}{(z-p)^{m}}+\frac{\phi_{1}}{(z-p)^{m-1}}+\cdots+\frac{\phi_{m-1}}{z-p} \in \mathcal{M}_{p}(E) .
$$

- Now let $E=C^{\infty}(Y)$. For $\phi, \psi \in C^{\infty}(Y)$, let $\phi \otimes \psi$ be the rank-one operator $C^{\infty}(Y) \ni h \mapsto(h, \psi) \phi \in C^{\infty}(Y)$. More generally, for $F, G \in \mathcal{M}_{p}\left(C^{\infty}(Y)\right)$, we introduce the meromorphic operator family $F(z) \otimes G(z)$ by

$$
F(z) \otimes G(z) h:=(h, G(\bar{z})) F(z), \quad h \in C^{\infty}(Y)
$$

where $(h, G(\bar{z}))=\langle h, \bar{G}(z)\rangle$ and $\bar{G}(z):=\overline{G(\bar{z})}$. For $\Phi, \Psi \in\left[C^{\infty}(Y)\right]^{\infty}$, we further set

$$
\begin{aligned}
& (\Phi \otimes \Psi)[z-p] \\
:= & \frac{\phi_{0} \otimes \psi_{0}}{(z-p)^{m}}+\frac{\phi_{0} \otimes \psi_{1}+\phi_{1} \otimes \psi_{0}}{(z-p)^{m-1}}+\cdots+\frac{\phi_{0} \otimes \psi_{m-1}+\cdots+\phi_{m-1} \otimes \psi_{0}}{z-p},
\end{aligned}
$$

where $\Phi=\left(\phi_{0}, \ldots, \phi_{m-1}\right), \Psi=\left(\psi_{0}, \ldots, \psi_{m-1}\right)$. In particular,

$$
(\Phi \otimes \Psi)[z-p]=(z-p)^{m}[\Phi[z-p] \otimes \Psi[z-p]]_{p}^{*},
$$

where $m=\max \{m(\Phi), m(\Psi)\}$.

- If $E$ is a Banach space, then we use the same notation, but with ( , ) replaced by the dual pairing $\langle$,$\rangle between E$ and $E^{\prime}$. This is due to the fact that in this situation we are working with the dual instead of the anti-dual to $E$. In particular, $\phi \otimes \psi$ for $\phi \in E, \psi \in E^{\prime}$ means the rank-one operator $E \ni h \rightarrow\langle h, \psi\rangle \phi \in E$, while (1.14), (1.15) are formally unchanged.

- On $\left[C^{\infty}(Y)\right]^{\infty}$, we introduce three commuting involutions by

$$
\begin{aligned}
\boldsymbol{C} \Phi & :=\left(\bar{\phi}_{0}, \bar{\phi}_{1}, \ldots, \bar{\phi}_{m-2}, \bar{\phi}_{m-1}\right), \\
\boldsymbol{I} \Phi & :=\left((-1)^{m} \bar{\phi}_{0},(-1)^{m-1} \bar{\phi}_{1}, \ldots, \bar{\phi}_{m-2},-\bar{\phi}_{m-1}\right), \\
\boldsymbol{J} \Phi & :=\left((-1)^{m} \phi_{0},(-1)^{m-1} \phi_{1}, \ldots, \phi_{m-2},-\phi_{m-1}\right),
\end{aligned}
$$

where $\Phi=\left(\phi_{0}, \phi_{1}, \ldots, \phi_{m-2}, \phi_{m-1}\right)$. Note that $\boldsymbol{I}=\boldsymbol{C} \boldsymbol{J}, \boldsymbol{T} \boldsymbol{C}=\boldsymbol{C T}, \boldsymbol{I} T+T \boldsymbol{I}=0$, and $\boldsymbol{J} T+T \boldsymbol{J}=0$.

- The cut-off function $\omega \in C_{\text {comp }}^{\infty}\left(\overline{\mathbb{R}}_{+}\right)$satisfies $\omega(t)=1$ for $t \leq 1 / 2, \omega(t)=0$ for $t \geq 1$. It is used to localize into the collar neighborhood $U \cong[0,1) \times Y$ of $\partial X$. 


\section{Discrete Asymptotic types}

The notion of a discrete asymptotic type for conormal cone asymptotics goes back to Rempel-Schulze [15] in the one-dimensional and Schulze [16] in the higher-dimensional case. It allows one to incorporate asymptotic information into a functional-analytic setting; see also Appendix B. The refinements of this notion presented here are due to LIU-WITT [12.

2.1. Preliminaries. Let $C_{\mathrm{as}}^{\infty, \delta}(X)$ be the space of all $u \in C^{\infty}\left(X^{\circ}\right)$ possessing an asymptotic expansion as in (1.5), as $x \rightarrow \partial X$, where, additionally, $\operatorname{Re} p<$ $\operatorname{dim} X / 2-\delta$ if $m_{p} \geq 1$. Moreover, let $C_{\mathcal{O}}^{\infty}(X)$ be the space of all $u \in C^{\infty}\left(X^{\circ}\right)$ vanishing to the infinite order on $\partial X$ (i.e., $\phi_{l}^{(p)}(y)=0$ for all $p, l$ in (1.5)).

Henceforth, we shall fix a splitting $U \backslash \partial X \cong(0,1) \times Y, x \mapsto(t, y)$ of coordinates near $\partial X$. However, it turns out that our constructions are independent of this splitting of coordinates; see Remarks 2.2, 2.5, and 2.11 as well as Proposition B.4.

Definition 2.1. (a) A discrete subset $V \subset \mathbb{C}$ is called a carrier of asymptotics if $|\operatorname{Re} p| \rightarrow \infty$ on $V$ as $|p| \rightarrow \infty$. For $\delta \in \mathbb{R}$, we write $V \in \mathcal{C}^{\delta}$ if, in addition, $V \subset\{z \in \mathbb{C} ; \operatorname{Re} z<\operatorname{dim} X / 2-\delta\}$.

(b) For $V \in \mathcal{C}^{\delta}$, we define $\mathcal{E}_{V}^{\delta}(Y)$ to be the space of all mappings $\Phi: \mathbb{C} \rightarrow$ $\left[C^{\infty}(Y)\right]^{\infty}$ satisfying $\{p \in \mathbb{C} \mid \Phi(p) \neq 0\} \subseteq V$. In particular, we have $\mathcal{E}_{V}^{\delta}(Y)=$ $\prod_{p \in V}\left[C^{\infty}(Y)\right]_{p}^{\infty}$, where $\left[C^{\infty}(Y)\right]_{p}^{\infty}$ is an isomorphic copy of $\left[C^{\infty}(Y)\right]^{\infty}$. Moreover, we set $\mathcal{E}^{\delta}(Y):=\bigcup_{V \in \mathcal{C}^{\delta}} \mathcal{E}_{V}^{\delta}(Y)$.

The operations $T, \boldsymbol{C}, \boldsymbol{I}$, and $\boldsymbol{J}$ are point-wise defined on $\mathcal{E}^{\delta}(Y)$; see Section 1.3 . For instance, $T \Phi(p)=T(\Phi(p))$ for $\Phi \in \mathcal{E}^{\delta}(Y), p \in \mathbb{C}$. We also write $m^{p}(\Phi)$ instead of $m(\Phi(p))$.

We next provide an isomorphism

$$
C_{\mathrm{as}}^{\infty, \delta}(X) / C_{\mathcal{O}}^{\infty}(X) \rightarrow \mathcal{E}^{\delta}(Y)
$$

that is non-canonical in the sense that it depends on the choice of splitting of coordinates near $\partial X$ : with the vector $\Phi \in \mathcal{E}_{V}^{\delta}(Y)$, where $\Phi(p)=\left(\phi_{0}^{(p)}, \ldots, \phi_{m_{p}-1}^{(p)}\right)$ for $p \in V$, we associate the formal asymptotic expansion

$$
u(x) \sim \sum_{p \in V} \sum_{k+l=m_{p}-1} \frac{(-1)^{k}}{k !} t^{-p} \log ^{k} t \phi_{l}^{(p)}(y) \text { as } t \rightarrow+0 .
$$

(To see that (2.1) is surjective uses a Borel-type argument.)

Remark 2.2. The operator $T$ acting on the quotient $C_{\text {as }}^{\infty, \delta}(X) / C_{\mathcal{O}}^{\infty}(X)-$ as introduced in (1.6) - is well-defined, i.e., it is independent of the chosen splitting of coordinates near $\partial X$. Moreover, the isomorphism (2.1) intertwines this operator and the right-shift operator $T$ acting on $\mathcal{E}^{\delta}(Y)$.

We need some further notation: for $\Phi \in \mathcal{E}^{\delta}(Y)$, we denote

$$
c-\operatorname{ord}(\Phi):=\operatorname{dim} X / 2-\max \{\operatorname{Re} p ; \Phi(p) \neq 0\}
$$

(the "conormal order" of $\Phi$ in an $L^{2}$-sense). Note that c-ord $(\Phi)>\delta$ if $\Phi \in \mathcal{E}^{\delta}(Y)$, and $\mathrm{c}-\operatorname{ord}\left(T^{k} \Phi\right) \rightarrow \infty$ as $k \rightarrow \infty$. Note also that, for $\Phi_{i} \in \mathcal{E}^{\delta}(Y), \alpha_{i} \in \mathbb{C}$ for $i=1,2, \ldots$ satisfying c-ord $\left(\Phi_{i}\right) \rightarrow \infty$ as $i \rightarrow \infty$, the series $\sum_{i=1}^{\infty} \alpha_{i} \Phi_{i}$ is an element 
of $\mathcal{E}^{\delta}(Y)$ in a natural manner. In particular,

$$
\sum_{i=1}^{\infty} \alpha_{i} \Phi_{i}=0 \quad \Longleftrightarrow \quad \operatorname{cord}\left(\sum_{i=1}^{i_{0}} \alpha_{i} \Phi_{i}\right) \rightarrow \infty \quad \text { as } i_{0} \rightarrow \infty .
$$

Furthermore, we need:

Definition 2.3. Let $\Phi \in \mathcal{E}^{\delta}(Y), \Phi \neq 0$. Then $\Phi$ is called a special vector if $\Phi \in \mathcal{E}_{p-\mathbb{N}_{0}}^{\delta}(Y)$ for some $p \in \mathbb{C}$. This $p$ is uniquely determined by the additional requirement that $\Phi(p) \neq 0$ and is then denoted by $\gamma(\Phi)$.

2.2. Definition of discrete asymptotic types. Discrete asymptotic types are certain linear subspace of the space $C_{\mathrm{as}}^{\infty, \delta}(X) / C_{\mathcal{O}}^{\infty}(X)$ of all formal asymptotic expansions.

Definition 2.4. (a) A discrete asymptotic type, $P$, for conormal cone asymptotics as $x \rightarrow \partial X$, of conormal order at least $\delta$, is a linear subspace of $C_{\mathrm{as}}^{\infty, \delta}(X) / C_{\mathcal{O}}^{\infty}(X)$ that via the isomorphism (2.1) is represented (in the given splitting $U \backslash \partial X \cong$ $(0,1) \times Y, x \mapsto(t, y)$ of coordinates near $\partial X)$ by a linear subspace $J \subset \mathcal{E}_{V}^{\delta}(Y)$ for some $V \in \mathcal{C}^{\delta}$ satisfying the following conditions:

(i) $T J \subseteq J$.

(ii) $\operatorname{dim} J^{\delta+j}<\infty$ for all $j \in \mathbb{N}_{0}$, where $J^{\delta^{\prime}}:=J /\left(J \cap \mathcal{E}^{\delta^{\prime}}(Y)\right)$ for $\delta^{\prime} \geq \delta$.

(iii) There is a sequence $\left\{p_{i} ; 1 \leq i<e+1\right\} \subset \mathbb{C}$, where $e \in \mathbb{N}_{0} \cup\{\infty\}$, such that $\operatorname{Re} p_{i}<\operatorname{dim} X / 2-\delta$ for all $i, \operatorname{Re} p_{i} \rightarrow-\infty$ as $i \rightarrow \infty$ if $e=\infty, V \subseteq \bigcup_{i=1}^{e}\left\{p_{i}\right\}-\mathbb{N}_{0}$, and

$$
J=\bigoplus_{i=1}^{e}\left(J \cap \mathcal{E}_{p_{i}-\mathbb{N}_{0}}^{\delta}(Y)\right)
$$

(b) The empty asymptotic type, $\mathcal{O}$, is represented by the trivial subspace $\{0\} \subset$ $\mathcal{E}^{\delta}(Y)$.

(c) The set of all asymptotic types of conormal order at least $\delta$ is denoted by $\underline{A s}^{\delta}(Y)$.

Remark 2.5. It can be shown that this notion of discrete asymptotic type is independent of the splitting of coordinates near $\partial X$. The latter means that changing coordinates $P \subset C_{\text {as }}^{\infty, \delta}(X) / C_{\mathcal{O}}^{\infty}(X)$ is represented by another linear subspace $J^{\prime} \subset \mathcal{E}_{V^{\prime}}^{\delta}(Y)$ for some $V^{\prime} \in \mathcal{C}^{\delta}$ also satisfying (i) to (iii) of Definition 2.4(a).

Let $P, P^{\prime} \in \underline{\mathrm{As}^{\delta}}$ be represented by $J, J^{\prime} \subset \mathcal{E}^{\delta}(Y)$, respectively. For $\delta^{\prime} \geq \delta$, we say that $P, P^{\prime}$ coincide up to the conormal order $\delta^{\prime}$ if $J^{\delta^{\prime}}=J^{\prime \delta^{\prime}}$ as subspaces of $\mathcal{E}^{\delta}(Y) / \mathcal{E}^{\delta^{\prime}}(Y)$. Similarly, for $\delta^{\prime}>\delta$, we say that $P, P^{\prime}$ coincide up to the conormal order $\delta^{\prime}-0$ if $P, P^{\prime}$ coincide up to the conormal order $\delta^{\prime}-\varepsilon$ for all $0<\varepsilon<\delta^{\prime}-\delta$.

It is important to observe that the set $\underline{A s}^{\delta}(X)$ of asymptotic types is partially ordered by inclusion of the representing spaces. This partial order on $\underline{\mathrm{As}^{\delta}}(X)$ will be denoted by $\preccurlyeq$. One of the fundamental principles in constructing asymptotic types obeying certain prescribed properties ensues from the following:

Proposition 2.6. $\left(\underline{\mathrm{As}}^{\delta}(X), \preccurlyeq\right)$ is a lattice with the property that each non-empty subset (resp. each bounded subset) possesses a greatest lower bound (resp. a least upper bound).

For example, consider $P \in \underline{\mathrm{As}^{\delta}}$ and let $\delta^{\prime} \geq \delta$. Then let $P^{\delta^{\prime}} \preccurlyeq P$ denote the largest asymptotic type that coincides with the empty asymptotic type, $\mathcal{O}$, up to 
the conormal order $\delta^{\prime}$. Similarly, for $\delta^{\prime}>\delta$, let $P^{\delta^{\prime}-0} \preccurlyeq P$ denote the largest asymptotic type that coincides with the empty asymptotic type up to the conormal order $\delta^{\prime}-0$. Of course, in these situations it is easy to provide representing spaces for $P^{\delta^{\prime}}$ and $P^{\delta^{\prime}-0}$, respectively, but in more involved situations this might be difficult.

Remark 2.7. There is an abstract concept of introducing asymptotic types if a unital algebra $\mathfrak{M}$ acting on some linear space $\mathfrak{F}$ "modulo a distinguished linear subspace $\mathfrak{F}_{0}$ in the image" is given. In our case, $\mathfrak{M}=\bigcup_{\mu \in \mathbb{Z}} \operatorname{Symb}_{M}^{\mu}(Y)$ is the algebra of complete Mellin symbols; see Section $3, \mathfrak{F}=C_{\text {as }}^{\infty, \delta}(X)$, and $\mathfrak{F}_{0}=C_{\mathcal{O}}^{\infty}(X)$. See WitT [19].

2.3. Proper discrete asymptotic types. Here, we investigate properties of linear subspaces $J \subset \mathcal{E}_{V}^{\delta}(Y)$ satisfying (i) to (iii) of Definition 2.4(a). This material is taken from LiU-WITT [12, Section 2.2].

Proposition 2.8. Let $J \subset \mathcal{E}_{V}^{\delta}(Y)$ be a linear subspace for some $V \in \mathcal{C}^{\delta}$. Then there are an $e \in \mathbb{N}_{0} \cup\{\infty\}$ and a sequence $\left\{\Phi_{i} ; 1 \leq i<e+1\right\}$ of special vectors satisfying c-ord $\left(\Phi_{i}\right) \rightarrow \infty$ as $i \rightarrow \infty$ if $e=\infty$, such that the vectors $T^{k} \Phi_{i}$ for $1 \leq i<e+1, k \in \mathbb{N}_{0}$ span the space $J$ if and only if (i) to (iii) of Definition 2.4(a) are fulfilled.

For the rest of this section, assume that $J \subset \mathcal{E}_{V}^{\delta}(Y)$ is a linear subspace satisfying (i) to (iii) of Definition 2.4 (a). Let $\Pi_{j}: J \rightarrow J^{\delta+j}$ be the canonical surjection. For $j^{\prime}>j$, there is a natural surjective mapping $\Pi_{j j^{\prime}}: J^{\delta+j^{\prime}} \rightarrow J^{\delta+j}$ such that $\Pi_{j j^{\prime \prime}}=\Pi_{j j^{\prime}} \Pi_{j^{\prime} j^{\prime \prime}}$ for $j^{\prime \prime}>j^{\prime}>j$ and

$$
\left(J, \Pi_{j}\right)=\lim _{\longleftarrow}\left(J^{\delta+j}, \Pi_{j j^{\prime}}\right) .
$$

Note that $T: J^{\delta+j} \rightarrow J^{\delta+j}$ is nilpotent, where the operator $T$ is induced by $T: J \rightarrow$ $J$. Let $\left(m_{1}^{j}, \ldots, m_{e_{j}}^{j}\right)$ be the characteristic of $J^{\delta+j}$; see (1.13) and thereafter.

The sequence $\left\{\Phi_{i} ; 1 \leq i<e+1\right\} \subset J$ is said to be a characteristic basis of $J$ if there are numbers $m_{i} \in \mathbb{N}_{0} \cup\{\infty\}, m_{i} \geq 1$, such that $T^{m_{i}} \Phi_{i}=0$ if $m_{i}<\infty$, while the sequence $\left\{T^{k} \Phi_{i} ; 1 \leq i<e+1,0 \leq k<m_{i}\right\}$ forms a basis of $J$.

Proposition 2.9. Let $J \subset \mathcal{E}_{V}^{\delta}(Y)$ be a linear subspace as above and assume that $\left\{\Phi_{i} ; 1 \leq i<e+1\right\}$ is a characteristic basis of $J$. Then the following conditions are equivalent:

(a) For each $j,\left\{\Pi_{j} \Phi_{1}, \ldots, \Pi_{j} \Phi_{e_{j}}\right\}$ is a characteristic basis of $J^{\delta+j}$.

(b) For each $j, T^{m_{1}^{j}-1} \Phi_{1}, \ldots, T^{m_{e_{j}}-1} \Phi_{e_{j}}$ are linearly independent over the space $\mathcal{E}^{\delta+j}(Y)$, while $T^{k} \Phi_{i} \in \mathcal{E}^{\delta+j}(Y)$, where either $1 \leq i \leq e_{j}, k \geq m_{i}^{j}$ or $i>e_{j}$.

If these conditions hold, then the numbering within the tuples $\left(m_{1}^{j}, \ldots, m_{e_{j}}^{j}\right)$ can be chosen in such a way that, for each $j \geq 1$, there is a characteristic basis $\Phi_{1}^{j}, \ldots, \Phi_{e_{j}}^{j}$ of $J^{\delta+j}$ such that, for all $j^{\prime}>j$,

$$
\Pi_{j j^{\prime}} \Phi_{i}^{j^{\prime}}= \begin{cases}\Phi_{i}^{j} & \text { if } 1 \leq i \leq e_{j} \\ 0 & \text { if } e_{j}+1 \leq i \leq e_{j^{\prime}}\end{cases}
$$


Furthermore, the scheme

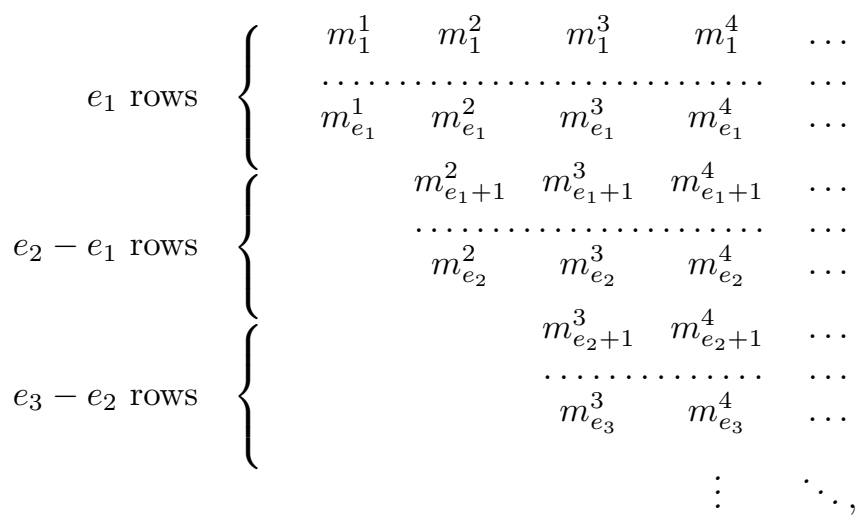

where in the $j$ th column the characteristic of the space $J^{\delta+j}$ appears, is uniquely determined up to permutation of the $k$ th and the $k^{\prime}$ th row, where $e_{j}+1 \leq k, k^{\prime} \leq$ $e_{j+1}$ for some $j\left(e_{0}=0\right)$.

Definition 2.10. An asymptotic type $P$ is said to be proper if its representing space $J$ possesses a characteristic basis $\left\{\Phi_{i} ; 1 \leq i<e+1\right\}$ consisting of special vectors that fulfil the equivalent conditions of Proposition [2.9. If the tuples $\left(m_{1}^{j}, \ldots, m_{e_{j}}^{j}\right)$ are re-ordered according to this proposition, then the sequence

$$
\left\{\left(\gamma\left(\Phi_{i}\right) ; m_{i}^{j_{i}}, m_{i}^{j_{i}+1}, m_{i}^{j_{i}+2}, \ldots\right) ; 1 \leq i<e+1\right\}
$$

is called the characteristic of $P$.

Remark 2.11. The characteristic of a proper asymptotic type $P \in \underline{A s}^{\delta}(Y)$ is independent of the splitting of coordinates near $\partial X$.

An asymptotic type need not be proper. For an example, see LiU-WitT [12, Example 2.23]. However, we have the following result, which will be generalized in Theorem 3.10 below:

Theorem 2.12. Let $A \in \operatorname{Diff}_{\text {cone }}^{\mu}(X)$ be an elliptic cone differential operator. Then

$$
\left\{u \in C_{\mathrm{as}}^{\infty, \delta}(X) \mid A u \in C_{\mathcal{O}}^{\infty}(X)\right\} / C_{\mathcal{O}}^{\infty}(X)
$$

is a proper asymptotic type.

\section{The Algebra of COMPlete Mellin symbols}

We study the algebra of complete Mellin symbols under the Mellin translation product. Furthermore, we introduce the important notion of a complete characteristic basis for the asymptotics annihilated by a holomorphic complete Mellin symbol.

3.1. Cone differential operators. Recall that we have fixed a splitting of coordinates $U \rightarrow[0,1) \times Y, x \mapsto(t, y)$ near $\partial X, U$ being a collar neighborhood of $\partial X$. Let $(\tau, \eta)$ be the covariables to $(t, y)$. The compressed covariable $t \tau$ to $t$ is denoted by $\tilde{\tau}$, i.e., $(\tilde{\tau}, \eta)$ is the linear variable in the fiber of the cone cotangent bundle $\tilde{T}^{*} U$, that is closely related to the $b$-cotangent bundle; see GiL-Krainer-Mendoza [3], Melrose [13]. 
For $A \in \operatorname{Diff}_{\text {cone }}^{\mu}(X)$ as given in (1.1), we denote by $\sigma_{\psi}^{\mu}(A)$ its principal symbol, by $\tilde{\sigma}_{\psi}^{\mu}(A)$ its compressed principal symbol defined on $\tilde{T}^{*} U$ by

$$
\sigma_{\psi}^{\mu}(A)(t, y, \tau, \eta)=t^{-\mu} \tilde{\sigma}_{\psi}^{\mu}(A)(t, y, t \tau, \eta), \quad(t, y, \tau, \eta) \in T^{*}(U \backslash \partial X) \backslash 0,
$$

and by $\sigma_{c}^{\mu-j}(A)(z)$ for $j=0,1,2, \ldots$ its $j$ th conormal symbol,

$$
\sigma_{c}^{\mu-j}(A)(z):=\sum_{k=0}^{\mu} \frac{1}{j !} \frac{\partial^{j} a_{k}}{\partial t^{j}}\left(0, y, D_{y}\right) z^{k}, \quad z \in \mathbb{C} .
$$

$\tilde{\sigma}_{\psi}^{\mu}(A)(t, y, \tilde{\tau}, \eta)$ is smooth up to $t=0$ and $\sigma_{c}^{\mu-j}(A)(z)$ for $j=0,1,2, \ldots$ is a holomorphic function in $z$ taking values in $\operatorname{Diff}^{\mu}(Y)$.

Furthermore, if $A \in \operatorname{Diff}_{\text {cone }}^{\mu}(X), B \in \operatorname{Diff}_{\text {cone }}^{\nu}(X)$, then $A B \in \operatorname{Diff}_{\text {cone }}^{\mu+\nu}(X)$ and

$$
\sigma_{c}^{\mu+\nu-l}(A B)(z)=\sum_{j+k=l} \sigma_{c}^{\mu-j}(A)(z+\nu-k) \sigma_{c}^{\nu-k}(B)(z)
$$

for $l=0,1,2, \ldots$ This formula is called the Mellin translation product.

Definition 3.1. (a) The operator $A \in \operatorname{Diff}_{\text {cone }}^{\mu}(X)$ is called elliptic if $A$ is an elliptic differential operator on $X^{\circ}$ and

$$
\tilde{\sigma}_{\psi}^{\mu}(A)(t, y, \tilde{\tau}, \eta) \neq 0, \quad(t, y, \tilde{\tau}, \eta) \in \tilde{T}^{*} U \backslash 0 .
$$

(b) The operator $A \in \operatorname{Diff}_{\text {cone }}^{\mu}(X)$ is called elliptic with respect to the weight $\delta \in \mathbb{R}$ if $A$ is elliptic in the sense of (a) and, in addition,

$$
\sigma_{c}^{\mu}(A)(z): H^{s}(Y) \rightarrow H^{s-\mu}(Y), \quad \operatorname{Re} z=\operatorname{dim} X / 2-\delta,
$$

is invertible for some $s \in \mathbb{R}$ (and then for all $s \in \mathbb{R}$ ).

Proposition 3.2. If $A \in \operatorname{Diff}_{\text {cone }}^{\mu}(X)$ is elliptic, then the set

$$
\left\{z \in \mathbb{C} \mid \sigma_{c}^{\mu}(A)(z) \text { regarded as operator in (3.3) is not invertible }\right\}
$$

is a carrier of asymptotics. In particular, there is a discrete set $D \subset \mathbb{R}$ such that $A$ is elliptic with respect to the weight $\delta$ for all $\delta \in \mathbb{R} \backslash D$.

3.2. Meromorphic Mellin symbols. We consider the class of meromorphic operator-valued functions arising in point-wise inverting elliptic conormal symbols $\sigma_{c}^{\mu}(A)(z)$. For further details, see SchulzE [17].

Definition 3.3. For $\mu \in \mathbb{Z} \cup\{-\infty\}$, the space $\mathcal{M}_{\text {as }}^{\mu}(Y)$ of Mellin symbols of order $\mu$ is defined as follows:

(a) The space $\mathcal{M}_{\mathcal{O}}^{\mu}(Y)$ of holomorphic Mellin symbols of order $\mu$ is the space of all $L_{\mathrm{cl}}^{\mu}(Y)$-valued holomorphic functions $\mathfrak{m}(z)$ on $\mathbb{C}$ such that $\left.\mathfrak{m}(z)\right|_{z=\beta+i \tau} \in$ $L_{\mathrm{cl}}^{\mu}\left(Y ; \mathbb{R}_{\tau}\right)$ uniformly in $\beta \in\left[\beta_{0}, \beta_{1}\right]$, where $-\infty<\beta_{0}<\beta_{1}<\infty$. Here, $L_{\mathrm{cl}}^{\mu}\left(Y ; \mathbb{R}_{\tau}\right)$ denotes the space of parameter-dependent classical pseudodifferential operators of order $\mu$ on $Y$, with parameter $\tau \in \mathbb{R}$.

(b) $\mathcal{M}_{\text {as }}^{-\infty}(Y)$ is the space of all meromorphic functions $\mathfrak{m}(z)$ on $\mathbb{C}$ taking values in $L^{-\infty}(Y)$ satisfying the following conditions:

(i) The Laurent expansion around each pole $z=p$ of $\mathfrak{m}(z)$ has the form

$$
\mathfrak{m}(z)=\frac{\mathfrak{m}_{0}}{(z-p)^{\nu}}+\frac{\mathfrak{m}_{1}}{(z-p)^{\nu-1}}+\cdots+\frac{\mathfrak{m}_{\nu-1}}{z-p}+\sum_{j \geq 0} \mathfrak{m}_{\nu+j}(z-p)^{j},
$$

where $\mathfrak{m}_{0}, \mathfrak{m}_{1}, \ldots, \mathfrak{m}_{\nu-1} \in L^{-\infty}(Y)$ are finite-rank operators. 
(ii) If the poles of $\mathfrak{m}(z)$ are numbered in a certain way, $p_{1}, p_{2}, p_{3}, \ldots$, then $\left|\operatorname{Re} p_{j}\right| \rightarrow \infty$ as $j \rightarrow \infty$ if the number of poles is infinite.

(iii) For any function $\chi(z) \in C^{\infty}(\mathbb{C})$ such that $\chi(z)=0$ if $\operatorname{dist}\left(z, \bigcup_{j}\left\{p_{j}\right\}\right) \leq 1 / 2$ and $\chi(z)=1$ if $\operatorname{dist}\left(z, \bigcup_{j}\left\{p_{j}\right\}\right) \geq 1$, we have $\left.\chi(z) \mathfrak{m}(z)\right|_{z=\beta+i \tau} \in L^{-\infty}\left(Y ; \mathbb{R}_{\tau}\right)$ uniformly in $\beta \in\left[\beta_{0}, \beta_{1}\right]$, where $-\infty<\beta_{0}<\beta_{1}<\infty$.

(c) We eventually set $\mathcal{M}_{\text {as }}^{\mu}(Y):=\mathcal{M}_{\mathcal{O}}^{\mu}(Y)+\mathcal{M}_{\text {as }}^{-\infty}(Y)$.

Write $\mathfrak{m} \in \mathcal{M}_{\text {as }}^{\mu}(Y)$ for $\mu \in \mathbb{Z}$ as $\mathfrak{m}(z)=\mathfrak{m}_{0}(z)+\mathfrak{m}_{1}(z)$, where $\mathfrak{m}_{0} \in \mathcal{M}_{\mathcal{O}}^{\mu}(Y), \mathfrak{m}_{1} \in$ $\mathcal{M}_{\text {as }}^{-\infty}(Y)$. Then the (parameter-dependent) principal symbol $\sigma_{\psi}^{\mu}\left(\left.\mathfrak{m}_{0}(z)\right|_{z=\beta+i \tau}\right) \in$ $S_{\mathrm{cl}}^{(\mu)}\left(\left(T^{*} Y \times \mathbb{R}_{\tau}\right) \backslash 0\right)$ is independent of the choice of the decomposition of $\mathfrak{m}(z)$ and also independent of $\beta \in \mathbb{R}$.

Definition 3.4. $\mathfrak{m} \in \mathcal{M}_{\text {as }}^{\mu}(Y)$ for $\mu \in \mathbb{Z}$ is called elliptic if $\sigma_{\psi}^{\mu}\left(\left.\mathfrak{m}_{0}(z)\right|_{z=\beta+i \tau}\right) \neq 0$ everywhere.

Proposition 3.5. (a) $\bigcup_{\mu \in \mathbb{Z}} \mathcal{M}_{\mathrm{as}}^{\mu}(Y)$ is a filtered algebra with respect to the pointwise product as multiplication.

(b) $\mathfrak{m} \in \mathcal{M}_{\text {as }}^{\mu}(Y)$ is invertible within this algebra, i.e., with its inverse belonging to $\mathcal{M}_{\mathrm{as}}^{-\mu}(Y)$, if and only if $\mathfrak{m}(z)$ is elliptic.

We further introduce the algebra $\operatorname{Symb}_{M}^{\mu}(Y)$ of complete Mellin symbols.

Definition 3.6. For $\mu \in \mathbb{Z} \cup\{-\infty\}$, the space $\operatorname{Symb}_{M}^{\mu}(Y)$ consists of all sequences $\mathfrak{S}^{\mu}=\left\{\mathfrak{s}^{\mu-j}(z) ; j \in \mathbb{N}_{0}\right\} \subset \mathcal{M}_{\text {as }}^{\mu}(Y)$. Moreover, an element $\mathfrak{S}^{\mu} \in \operatorname{Symb}_{M}^{\mu}(Y)$ is called holomorphic if $\mathfrak{S}^{\mu}=\left\{\mathfrak{s}^{\mu-j}(z) ; j \in \mathbb{N}_{0}\right\} \subset \mathcal{M}_{\mathcal{O}}^{\mu}(Y)$.

Proposition 3.7. (a) $\bigcup_{\mu \in \mathbb{Z}} \operatorname{Symb}_{M}^{\mu}(Y)$ is a filtered algebra with involution with respect to the following operations:

(i) The Mellin translation product

$$
\operatorname{Symb}_{M}^{\mu}(Y) \times \operatorname{Symb}_{M}^{\nu}(Y) \rightarrow \operatorname{Symb}_{M}^{\mu+\nu}(Y), \quad\left(\mathfrak{S}^{\mu}, \mathfrak{T}^{\nu}\right) \mapsto \mathfrak{S}^{\mu} \circ_{M} \mathfrak{T}^{\nu},
$$

where $\mathfrak{S}^{\mu}=\left\{\mathfrak{s}^{\mu-j}(z) ; j \in \mathbb{N}_{0}\right\}, \mathfrak{T}^{\nu}=\left\{\mathfrak{t}^{\nu-k}(z) ; k \in \mathbb{N}_{0}\right\}, \mathfrak{S}^{\mu} \circ_{M} \mathfrak{T}^{\nu}=\left\{\mathfrak{u}^{\mu+\nu-l}(z)\right.$; $\left.l \in \mathbb{N}_{0}\right\}$, and

$$
\mathfrak{u}^{\mu+\nu-l}(z)=\sum_{j+k=l} \mathfrak{s}^{\mu-j}(z+\nu-k) \mathfrak{t}^{\nu-k}(z), \quad l=0,1,2, \ldots
$$

as multiplication.

(ii) The operation

$$
\operatorname{Symb}_{M}^{\mu}(Y) \rightarrow \operatorname{Symb}_{M}^{\mu}(Y), \quad \mathfrak{S}^{\mu} \mapsto\left(\mathfrak{S}^{\mu}\right)^{* M},
$$

where $\mathfrak{S}^{\mu}=\left\{\mathfrak{s}^{\mu-j}(z) ; j \in \mathbb{N}_{0}\right\},\left(\mathfrak{S}^{\mu}\right)^{*_{M}}=\left\{\mathfrak{r}^{\mu-j}(z) ; j \in \mathbb{N}_{0}\right\}$, and

$$
\mathfrak{r}^{\mu-j}(z)=\mathfrak{s}^{\mu-j}(\operatorname{dim} X-2 \delta-\bar{z}-\mu+j)^{*}, \quad j=0,1,2, \ldots,
$$

as involution.

(b) The complete Mellin symbol $\left\{\mathfrak{s}^{\mu-j}(z) ; j \in \mathbb{N}_{0}\right\} \in \operatorname{Symb}_{M}^{\mu}(Y)$ is invertible within the filtered algebra $\bigcup_{\mu \in \mathbb{Z}} \operatorname{Symb}_{M}^{\mu}(Y)$, i.e., with its inverse belonging to $\operatorname{Symb}_{M}^{-\mu}(Y)$, if and only if $\mathfrak{s}^{\mu}(z)$ is elliptic in the sense of Definition 3.4 .

(c) The map

$$
\bigcup_{\mu \in \mathbb{N}_{0}} \operatorname{Diff}_{\text {cone }}^{\mu}(X) \rightarrow \bigcup_{\mu \in \mathbb{Z}} \operatorname{Symb}_{M}^{\mu}(Y), \quad A \mapsto\left\{\sigma_{c}^{\mu-j}(A) ; j \in \mathbb{N}_{0}\right\},
$$

is a homomorphism of filtered algebras with involution. 
3.3. The space $L_{\mathfrak{S}^{\mu}}^{\delta}$. For a complete Mellin symbol $\mathfrak{S}^{\mu}$, we introduce a special notation for the representing space of the "asymptotics annihilated" by $\mathfrak{S}^{\mu}$. We actually restrict ourselves to the holomorphic complete Mellin symbol (although the definition can be generalized to the meromorphic complete Mellin symbol by taking into account the possible "production of asymptotics;" see LiU-WitT [12]). The reason is that Theorem 3.10, in general, fails to hold without assuming holomorphy.

Definition 3.8. (a) Let $\mathfrak{S}^{\mu} \in \operatorname{Symb}_{M}^{\mu}(Y)$ be holomorphic. Then $L_{\mathfrak{S}^{\mu}}^{\delta} \subset \mathcal{E}^{\delta}(Y)$ is the linear space spanned by all special vectors $\Phi \in \mathcal{E}^{\delta}(Y)$ satisfying

$$
\sum_{j+k=l} \mathfrak{s}^{\mu-k}(z+k) \Phi(p-j)[z-p+l] \in \mathcal{A}_{p-l}\left(C^{\infty}(Y)\right)
$$

for $l=0,1,2, \ldots$, where $p=\gamma(\Phi)$.

(b) In this situation described in Proposition 3.9 below, we will also write $L_{A}^{\delta}$ instead of $L_{\mathfrak{S}^{\mu}}^{\delta}$.

For later use, we introduce a notation for the expression appearing on the lefthand side of (3.8):

$$
\boldsymbol{\Theta}_{l}(\Phi)[z]=\boldsymbol{\Theta}_{l}\left(\Phi ; \mathfrak{S}^{\mu}\right)[z]:=\sum_{j+k=l} \mathfrak{s}^{\mu-k}(z+k) \Phi(p-j)[z-p+l] .
$$

Proposition 3.9. For $A \in \operatorname{Diff}_{\text {cone }}^{\mu}(Y)$, the subspace of $C_{\mathrm{as}}^{\infty, \delta}(Y) / C_{\mathcal{O}}^{\infty}(X)$ from (2.4) is represented by the linear space $L_{\mathfrak{S}^{\mu}}^{\delta}$, where $\mathfrak{S}^{\mu}=\left\{\sigma_{c}^{\mu-j}(A)(z) ; j \in \mathbb{N}_{0}\right\}$.

Proof. It is readily seen that the representing space $L \subset \mathcal{E}^{\delta}(Y)$ of the space $\{u \in$ $\left.C_{\mathrm{as}}^{\infty, \delta}(Y) \mid A u \in C_{\mathcal{O}}^{\infty}(X)\right\} / C_{\mathcal{O}}^{\infty}(X)$ from (2.4) satisfies conditions (i) to (iii) of Definition 2.4(a). Hence, by virtue of Proposition 2.8, $L$ is spanned by special vectors $\Phi \in \mathcal{E}^{\delta}(Y)$.

To show that $L=L_{\mathfrak{S}^{\mu}}^{\delta}$, it therefore suffices to prove that a special vector $\Phi \in$ $\mathcal{E}^{\delta}(Y)$ belongs to $L$ if and only if it satisfies condition (3.8). Pick a special vector $\Phi \in \mathcal{E}^{\delta}(Y)$, where

$$
\Phi(p-j)=\left(\phi_{0}^{(j)}, \phi_{1}^{(j)} \ldots, \phi_{m_{j}-1}^{(j)}\right), \quad j=0,1,2, \ldots,
$$

$p=\gamma(\Phi)$, and pick a function $u \in C_{\mathrm{as}}^{\infty, \delta}(Y)$ (say, of bounded support) such that

$$
u(x) \sim \sum_{j \geq 0} \sum_{r+s=m_{j}-1} \frac{(-1)^{r}}{r !} t^{-p+j} \log ^{r} t \phi_{s}^{(j)}(y) \quad \text { as } t \rightarrow+0 .
$$

The following computations are performed on an informal level; strict justification can be provided utilizing ideas from Schulze's cone algebra; see Schulze [16, 17]. We have

$$
A u(x) \sim \sum_{k \geq 0} t^{-\mu+k}\left[\operatorname{op}_{M}^{\delta}\left(\sigma_{c}^{\mu-k}(A)(z)\right) u\right](t, y) \quad \text { as } t \rightarrow+0,
$$

where the asymptotics are in increasing flatness as $t \rightarrow+0$. Here, for any $\mathfrak{s} \in$ $\mathcal{M}_{\text {as }}^{\mu}(Y)$,

$$
\begin{aligned}
{\left[\operatorname{op}_{M}^{\delta}(\mathfrak{s}(z)) u\right](t, y) } & =M_{z \rightarrow t}^{-1}\left\{\mathfrak{s}\left(z, y, D_{y}\right)(M u)(z, y)\right\} \\
& =\frac{1}{2 \pi i} \int_{\operatorname{Re} z=\operatorname{dim} X / 2-\delta} \int_{0}^{\infty}\left(\frac{t}{t^{\prime}}\right)^{-z} \mathfrak{s}\left(z, y, D_{y}\right) u\left(t^{\prime}, y\right) \frac{d t^{\prime}}{t^{\prime}} d z
\end{aligned}
$$


the latter understood as an oscillatory integral, and $M=M_{t \rightarrow z}\{\ldots\}$ is the Mellin transformation with respect to $t$. Writing $\tilde{u}(z)=M u(z, y)$, we get

$$
(A u)^{\sim}(z) \sim \sum_{k \geq 0} \sigma_{c}^{\mu-k}(A)(z-\mu+k) \tilde{u}(z-\mu+k)
$$

where now the asymptotics are in improving control of the meromorphic structure of $(A u)^{\sim}(z)$ : the addition of the $(k+1)$ th summand $\sigma_{c}^{\mu-k}(A)(z-\mu+k) \tilde{u}(z-\mu+k)$ for $k=0,1,2, \ldots$ provides control in a right-half plane up to $\operatorname{Re} z>\operatorname{dim} X / 2+\mu-$ $k-\delta-1$.

In fact, the only possible poles of $(A u)^{\sim}(z)$ occur at $p+\mu-l$ for $l=0,1,2, \ldots$, and

$$
\left[(A u)^{\sim}(z)\right]_{p+\mu-l}^{*}=\sum_{j+k=l}\left[\sigma_{c}^{\mu-k}(A)(z-\mu+k) \Phi(p-j)[z-p-\mu+l]\right]_{p+\mu-l}^{*}
$$

because of $[\tilde{u}(z-\mu+k)]_{p+\mu-l}^{*}=\Phi(p-j)[z-p-\mu+l]$ for $j+k=l$ by construction and $\sigma_{c}^{\mu-k}(A)(z-\mu+k)$ is holomorphic, i.e.,

$$
\left[(A u)^{\sim}(z+\mu)\right]_{p-l}^{*}=\sum_{j+k=l}\left[\sigma_{c}^{\mu-k}(A)(z+k) \Phi(p-j)[z-p+l]\right]_{p-l}^{*} .
$$

Now, $A u \in C_{\mathcal{O}}^{\infty}(X)$ if and only if $(A u)^{\sim}(z+\mu)$ is holomorphic in the whole of $\mathbb{C}$. Then this yields condition (3.8), with $\mathfrak{s}^{\mu-k}(z)$ replaced by $\sigma_{c}^{\mu-k}(A)(z)$.

Generalizing Theorem 2.12, we have:

Theorem 3.10 (LiU-WITT [12, Theorem 2.31]). For an elliptic, holomorphic $\mathfrak{S}^{\mu} \in$ $\operatorname{Symb}_{M}^{\mu}(Y), L_{\mathfrak{S}^{\mu}}^{\delta}$ represents a proper asymptotic type.

In proof of Theorem 4.1, we need:

Lemma 3.11. The adjoint relation to (3.8),

$$
\sum_{j+k=l} \mathfrak{r}^{\mu-k}(z+k) \Psi(q-j)[z-q+l] \in \mathcal{A}_{q-l}\left(C^{\infty}(Y)\right)
$$

where $\mathfrak{R}^{\mu} \in \operatorname{Symb}_{M}^{\mu}(Y)$ is as in $(\underline{3.6}$, is equivalent to

$$
\sum_{j+k=l} \mathfrak{s}^{\mu-k}(z)^{t} \boldsymbol{I} \Psi(q-j)[z-p-l] \in \mathcal{A}_{p+l}\left(C^{\infty}(Y)\right)
$$

where $q=\operatorname{dim} X-2 \delta-\bar{p}-\mu$.

3.4. Complete characteristic bases. The control of asymptotics of the form (1.5), of conormal order at least $\delta$, is equivalent to the control of the conormal symbols $\sigma_{c}^{\mu-j}(A)(z)$ for $j=0,1,2, \ldots$ in the half spaces $\operatorname{Re} z<\operatorname{dim} X / 2-\delta-j$. We now investigate what happens as $\delta \rightarrow-\infty$.

Let

$$
\mathcal{E}(Y):=\bigcup_{\delta \in \mathbb{R}} \mathcal{E}^{\delta}(Y),
$$

and $L_{\mathfrak{S}^{\mu}}:=\bigcup_{\delta \in \mathbb{R}} L_{\mathfrak{S}^{\mu}}^{\delta}$ for a holomorphic $\mathfrak{S}^{\mu} \in \operatorname{Symb}_{M}^{\mu}(Y)$. 
Definition 3.12. A complete characteristic basis of $L_{\mathfrak{S}^{\mu}}$ is the inductive limit

$$
\lim _{\longrightarrow}\left(\left\{\Phi_{h}^{\delta} ; h \in \mathcal{I}^{\delta}\right\}, \tau_{\delta^{\prime} \delta}\right)
$$

of the following inductive system:

(a) For each $\delta \in \mathbb{R},\left\{\Phi_{h}^{\delta} ; h \in \mathcal{I}^{\delta}\right\}$ is a characteristic basis of $L_{\mathfrak{S}^{\mu}}^{\delta}$ of characteristic $\left(\gamma\left(\Phi_{h}^{\delta}\right) ; m_{h}^{j_{h}, \delta}, m_{h}^{j_{h}+1, \delta}, \ldots\right)$ satisfying conditions (a), (b) of Proposition 2.9.

(b) For all $\delta>\delta^{\prime}, \tau_{\delta^{\prime} \delta}: \mathcal{I}^{\delta} \rightarrow \mathcal{I}^{\delta^{\prime}}$ is an injection such that, for any $h \in \mathcal{I}^{\delta}$,

(i) $\gamma\left(\Phi_{h^{\prime}}^{\delta^{\prime}}\right)=\gamma\left(\Phi_{h}^{\delta}\right)+a$ for some $a \in \mathbb{N}_{0}$,

(ii) $\Phi_{h}^{\delta}=T_{h^{\prime}}^{m_{h^{\prime}}+a-1, \delta^{\prime}} \Phi_{h^{\prime}}^{\delta^{\prime}}$,

where $h^{\prime}=\tau_{\delta^{\prime} \delta}(h)$ (and $\tau_{\delta^{\prime \prime} \delta}=\tau_{\delta^{\prime \prime} \delta^{\prime}} \tau_{\delta^{\prime} \delta}$ for $\left.\delta>\delta^{\prime}>\delta^{\prime \prime}\right)$.

We write $\mathcal{I}:=\lim \left(\mathcal{I}^{\delta}, \tau_{\delta^{\prime} \delta}\right)$ with injections $\tau_{\delta}: \mathcal{I}^{\delta} \rightarrow \mathcal{I}$ (such that $\tau_{\delta}=\tau_{\delta^{\prime}} \tau_{\delta^{\prime} \delta}$ for $\delta>\delta^{\prime}$ ) and

$$
\left\{\Phi_{h} ; h \in \mathcal{I}\right\}=\underline{\lim }\left(\left\{\Phi_{h}^{\delta} ; h \in \mathcal{I}^{\delta}\right\}, \tau_{\delta^{\prime} \delta}\right),
$$

where each $\Phi_{h}$ for $h \in \mathcal{I}$ is the collection $\left\{\Phi_{h^{\delta}}^{\delta}\right\}$ with $h=\left\{h^{\delta}\right\}$.

The proof of (a) in the next proposition relies on the finite-dimensionality of the spaces $J^{\delta+j}$ :

Proposition 3.13. (a) For each holomorphic $\mathfrak{S}^{\mu} \in \operatorname{Symb}_{M}^{\mu}(Y), L_{\mathfrak{S}^{\mu}}$ possesses a complete characteristic basis.

(b) For any complete characteristic basis $\left\{\Phi_{h} ; h \in \mathcal{I}\right\}$ of $L_{\mathfrak{S}^{\mu}}$, the expression

$$
T^{m^{p+l}\left(\Phi_{h}\right)} \Phi_{h}(p):=T^{m^{p+l}\left(\Phi_{h}^{\delta} \delta\right.} \Phi_{h^{\delta}}^{\delta}(p),
$$

where $p \in \mathbb{C}, l \in \mathbb{N}_{0}, h^{\delta}=\tau_{\delta}(h)$, and $\delta$ is chosen in such a way that

$$
\delta \leq \max \left\{\min \left\{\delta^{\prime} \in \mathbb{R} \mid \gamma\left(\Phi_{h^{\delta^{\prime}}}^{\delta^{\prime}}\right)-\operatorname{Re} p \in \mathbb{N}_{0}\right\}, \operatorname{dim} X / 2-(\operatorname{Re} p+l)\right\},
$$

is well-defined. (If there is no $\delta$ such that $\gamma\left(\Phi_{h^{\delta}}^{\delta}\right)-\operatorname{Re} p \in \mathbb{N}_{0}$, then we set $T^{m^{p+l}\left(\Phi_{h}\right)} \Phi_{h}(p):=0$.)

\section{Singularity Structure of InVERSES}

In the sequel, let $\mathfrak{S}^{\mu}=\left\{\mathfrak{s}^{\mu-j}(z) ; j \in \mathbb{N}_{0}\right\} \in \operatorname{Symb}_{c}^{\mu}(Y)$ be holomorphic and elliptic. The inverse to $\mathfrak{S}^{\mu}$ will then be denoted by $\mathfrak{T}^{-\mu}=\left\{\mathfrak{t}^{-\mu-k}(z) ; k \in \mathbb{N}_{0}\right\} \in$ $\operatorname{Symb}_{M}^{-\mu}(Y)$; see Proposition 3.7 (b). In particular,

$$
\sum_{j+k=l} \mathfrak{t}^{-\mu-j}(z+\mu+j) \mathfrak{s}^{\mu-k}(z+l)=\delta_{0 l} \text { id }, \quad l=0,1,2, \ldots
$$

Before stating Theorems 4.1 and 5.1 we simplify the situation to be considered in their proofs. Due to the facts that

- in the process of inverting $\mathfrak{S}^{\mu}$ with respect to the Mellin translation product, the "production of singularities" of $\mathfrak{t}^{-\mu-j}(z+\mu)$ at $z=p$ and $\mathfrak{t}^{-\mu-j^{\prime}}(z+\mu)$ at $z=p^{\prime}$, respectively, influences each other only if $p-p^{\prime} \in \mathbb{Z}$,

- control of the singularity structure of $\mathfrak{t}^{-\mu-j}(z+\mu)$ in the half spaces $\operatorname{Re} z<$ $\operatorname{dim} X / 2-\delta$ for each $\delta \in \mathbb{R}$ provides control of the singularity structure of $\mathfrak{t}^{-\mu-j}(z+\mu)$ in the whole of $\mathbb{C}$,

we are allowed to assume the following model situation: the complete characteristic basis of $L_{\mathfrak{S}^{\mu}}$ consists of special vectors $\Phi_{i}$ for $1 \leq i<e+1$, where $e \in \mathbb{N}_{0} \cup\{\infty\}$, $\gamma\left(\Phi_{1}\right)=p$, and

$$
\gamma\left(\Phi_{i}\right)=p-l, \quad e_{l}+1 \leq i \leq e_{l+1}
$$


see Definition 2.3. Then $0=e_{0}<e_{1} \leq e_{2} \leq \ldots$ and $e=\max \left\{e_{l} ; l \in \mathbb{N}_{0}\right\}$. When referring to this model situation, we denote

$$
m_{i}^{l+1}:=m^{p-l}\left(\Phi_{i}\right) .
$$

Theorem 4.1. Let $\mathfrak{S}^{\mu} \in \operatorname{Symb}_{c}^{\mu}(Y)$ be elliptic and holomorphic. Then, for each complete characteristic basis $\left\{\Phi_{h} ; h \in \mathcal{I}\right\}$ of $L_{\mathfrak{S}^{\mu}}$, there is a unique complete characteristic basis $\left\{\Psi_{h^{*}} ; h^{*} \in \mathcal{I}^{*}\right\}$ of $L_{\Re^{\mu}}$, where $\mathfrak{R}^{\mu}$ is given by (3.6), and a bijection $\tau^{*}: \mathcal{I} \rightarrow \mathcal{I}^{*}$ such that, for all $p \in \mathbb{C}$ and $j=0,1,2, \ldots$,

$$
\left[\mathfrak{t}^{-\mu-j}(z+\mu)\right]_{p}^{*}=\sum_{h} T^{m_{h}^{p+1}} \Phi_{h}(p-j) \otimes T^{m_{h^{*}}^{q+j+1}} \boldsymbol{J} \Psi_{h^{*}}(q)[z-p],
$$

where $q=\operatorname{dim} X-2 \delta-\bar{p}-\mu, h^{*}=\tau^{*}(h), m_{h}^{p+1}=m^{p+1}\left(\Phi_{h}\right)$, and $m_{h^{*}}^{q+j+1}=$ $m^{q+j+1}\left(\Psi_{h^{*}}\right)$.

Proof. We assume the model situation (4.1).

Step 1. The elements $\Phi$ of $L_{\mathfrak{S}^{\mu}}\left(=L_{\mathfrak{S}^{\mu}}^{\delta}\right)$ are given by the relations

$$
\Phi(p-l)[z-p+l]=\sum_{j=0}^{l}\left[\mathfrak{t}^{-\mu-j}(z+\mu) \phi^{(p-l+j)}(z)\right]_{p-l+j}^{*},
$$

for $l=0,1,2, \ldots$, where $\phi^{(p-j)}=\sum_{r=0}^{\infty} \phi_{r}^{(p-j)}(z-p+j)^{r} \in \mathcal{A}_{p-j}\left(C^{\infty}(Y)\right)$ for $j=0,1,2, \ldots$ See LiU-WitT [12. In fact, the Taylor coefficients $\phi_{r}^{(p-j)} \in C^{\infty}(Y)$ can be chosen arbitrarily, since only a finite number of them enters the computation of $\Phi(p-l)$.

Since $\Phi(p-l) \in \operatorname{span}\left\{\Phi_{i}(p-l) ; 1 \leq i \leq e_{l+1}\right\}$ for $\Phi \in L_{\mathfrak{S}^{\mu}}$, we conclude that

$$
\left[\mathfrak{t}^{-\mu-j}(z+\mu)\right]_{p-l+j}^{*}=\sum_{i=1}^{e_{l+1}} \Phi_{i}(p-l) \otimes H_{i}^{(j l)}[z-p+l-j]
$$

for certain $H_{i}^{(j l)}=\left(h_{0 i}^{(j l)}, h_{1 i}^{(j l)}, \ldots, h_{m_{i}^{l+1}-1, i}^{(j l)}\right) \in\left[C^{\infty}(Y)\right]^{\infty}$, which a priori are of length $m_{i}^{l+1}$. Employing (4.4), we rewrite (4.3) as

$$
\Phi(p-l)=\sum_{i=1}^{e_{l+1}} \sum_{r=0}^{m_{i}^{l+1}-1}\left(\sum_{j=0}^{l} \sum_{s=0}^{r}\left(\phi_{r-s}^{(p-l+j)}, h_{s i}^{(j l)}\right)\right) T^{r} \Phi_{i}(p-l)
$$

for $l=0,1,2, \ldots$.

Step 2. We are going to show that (4.5) provides the unique representation of $\Phi$ as a linear combination of the vectors $T^{r} \Phi_{i}$. More precisely, by induction on $l=0,1,2, \ldots$, we construct functions $h_{r i}^{(l)} \in C^{\infty}(Y)$ for $r \geq m_{i}^{l}$ such that, for each $l$,

$$
h_{m_{i}^{l}, i}^{(l)} \text { for all } i \text { satisfying } m_{i}^{l}<m_{i}^{l+1} \text { are linearly independent }
$$

and

$$
h_{r i}^{(j l)}= \begin{cases}h_{r i}^{(l-j)} & \text { if } r \geq m_{i}^{l-j}, \\ 0 & \text { otherwise. }\end{cases}
$$

This means that the coefficient in front of $T^{r} \Phi_{i}$ equals $\sum_{j=0}^{l} \sum_{s=m_{i}^{j}}^{r}\left(\phi_{r-s}^{(p-j)}, h_{s i}^{(j)}\right)$ provided that $m_{i}^{l} \leq r \leq m_{i}^{l+1}-1$. 
After the $l$ th step, $h_{r i}^{\left(l^{\prime}\right)}$ will have been constructed for all $l^{\prime}, i, r$ satisfying $l^{\prime} \leq l$, $1 \leq i \leq e_{l+1}, m_{i}^{l^{\prime}} \leq r \leq m_{i}^{l+1}-1$. Moreover, (4.7) will have been proved for all $i, r$ satisfying $1 \leq i \leq e_{l+1}, 0 \leq r \leq m_{i}^{l+1}-1$.

Base of induction $\boldsymbol{l}=0$ : We set $h_{r i}^{(0)}:=h_{r i}^{(00)}$ for $1 \leq i \leq e_{1}, 0 \leq r \leq m_{i}^{1}-1$.

Induction step $\boldsymbol{l}^{\prime}<\boldsymbol{l} \rightarrow \boldsymbol{l}$ : We write (4.5) as

$$
\begin{aligned}
\Phi(p-l)=\sum_{i=1}^{e_{l}} \sum_{r=0}^{m_{i}^{l}-1}\left(\sum_{j=0}^{l} \sum_{s=0}^{r}\left(\phi_{r-s}^{(p-l+j)}, h_{s i}^{(j l)}\right)\right) T^{r} \Phi_{i}(p-l) \\
\quad+\sum_{i=1}^{e_{l+1}} \sum_{r=m_{i}^{l}}^{m_{i}^{l+1}-1}\left(\sum_{j=0}^{l} \sum_{s=0}^{r}\left(\phi_{r-s}^{(p-l+j)}, h_{s i}^{(j l)}\right)\right) T^{r} \Phi_{i}(p-l) .
\end{aligned}
$$

$\Phi$ regarded as a vector in $L_{\mathfrak{S}^{\mu}}^{\delta}$ modulo $L_{\mathfrak{S}^{\mu}}^{\delta^{\prime}}$ for some $\delta^{\prime}$ satisfying $\operatorname{dim} X / 2-\delta^{\prime}<$ $p-l<\operatorname{dim} X / 2-\delta^{\prime}+1$ is a unique linear combination of the vectors $T^{r} \Phi_{i}$ for $1 \leq i \leq e_{l+1}, 0 \leq r \leq m_{i}^{l+1}-1$. The coefficient in front of $T^{r} \Phi_{i}$ for $1 \leq i \leq e_{l}$, $0 \leq r \leq m_{i}^{l}-1$, is known if one knows $\Phi$ modulo $L_{\mathfrak{S}^{\mu}}^{\delta^{\prime}-1}$. By inductive hypothesis, this coefficient equals $\sum_{j=0}^{l-1} \sum_{s=m_{i}^{j}}^{r}\left(\phi_{r-s}^{(p-j)}, h_{s i}^{(j)}\right)$. Thus, we obtain (4.7) for $1 \leq i \leq e_{l}$, $0 \leq r \leq m_{i}^{l}-1$, since the functions $\phi_{r}^{(p-j)} \in C^{\infty}(Y)$ are arbitrary.

It remains to set $h_{r i}^{\left(l^{\prime}\right)}:=h_{r i}^{\left(l-l^{\prime}, l\right)}$ for $0 \leq l^{\prime} \leq l, m_{i}^{l} \leq r \leq m_{i}^{l+1}-1$.

In particular,

$$
H_{i}^{(j l)}=\left(h_{m_{i}^{l-j}, i}^{(l-j)}, h_{m_{i}^{l-j}+1 i}^{(l-j)}, \ldots, h_{m_{i}^{l+1}-1, i}^{(l-j)}\right)
$$

is actually of length $m_{i}^{l+1}-m_{i}^{l-j}$.

Step 3. We now fix some $a \in \mathbb{N}_{0}$ and set

$$
H_{i}^{(l)}=H_{i ; a}^{(l)}:=\left(h_{m_{i}^{l}, i}^{(l)}, h_{m_{i}^{l-j}+1 i}^{(l)}, \ldots, h_{m_{i}^{a}-1, i}^{(l)}\right)
$$

for $0 \leq l \leq a-1$. Then $H_{i}^{(j l)}=T^{m_{i}^{a}-m_{i}^{l+1}} H_{i}^{(l-j)}$ and

$$
\begin{aligned}
& {\left[\mathfrak{t}^{-\mu-j}(z+\mu-k)\right]_{p-b}^{*}} \\
& \quad=\sum_{i=1}^{e_{l+b+1}} T^{m_{i}^{k+b}} \Phi_{i}(p-l-b) \otimes T^{m_{i}^{a}-m_{i}^{l+b+1}} H_{i}^{(k+b)}[z-p+b]
\end{aligned}
$$

for all $j, k, l, b$ satisfying $j+k=l, l+b<a$. We shall employ (4.8) to show that the vectors $\Psi_{1}, \ldots, \Psi_{e_{a}}$ defined by

$$
\Psi_{i}(q+l):=\boldsymbol{J} H_{i}^{(l)}, \quad 0 \leq l \leq a-1,
$$

where $q=\operatorname{dim} X-2 \delta-\bar{p}-\mu$, form a characteristic basis of $L_{\mathfrak{R}^{\mu}}^{\delta+\mu-a}$ modulo $L_{\mathfrak{R}^{\mu}}^{\delta+\mu}$.

In view of (4.6) $, \Psi_{1}, \ldots, \Psi_{e_{a}}$ form a characteristic basis of the $T$-invariant subspace of $\mathcal{E}^{\delta}(Y)$ modulo $\mathcal{E}^{\delta+a}(Y)$ generated by these vectors, of characteristic

$$
\left\{\left(q+l ; m_{i}^{a}-m_{i}^{l}, m_{i}^{a}-m_{i}^{l-1}, \ldots, m_{i}^{a}-m_{i}^{1}, m_{i}^{a}\right) ; 1 \leq i \leq e_{a}\right\},
$$

where, for a given $i, l$ is the least integer such that $m_{i}^{l+1}=m_{i}^{a}$. In particular, the dimension of this space equals $\operatorname{dim} L_{\mathfrak{S}^{\mu}}^{\delta} / L_{\mathfrak{S}^{\mu}}^{\delta+a}=\sum_{i=1}^{e_{a}} m_{i}^{a}$. Invoking a duality argument, we see that it suffices to prove that each $\Psi_{i}$ belongs to $L_{\mathfrak{R}^{\mu}}^{\delta+\mu-a}$ modulo $L_{\mathfrak{R}^{\mu}}^{\delta+\mu}$. 
Step 4. By virtue of (3.10), we have to show that

$$
\sum_{j+k=l} \mathfrak{s}^{\mu-k}(z)^{t} \boldsymbol{C} H_{i}^{(a-j-1)}[z-\tilde{p}-l]=O(1) \quad \text { as } z \rightarrow \tilde{p}+l
$$

for $1 \leq i \leq e_{a}, 0 \leq l \leq a-1$, where $\tilde{p}:=p-a+1$.

For $l+b=a-1$, we infer from (4.8)

$$
\begin{aligned}
& \delta_{0 l} \text { id }=\sum_{j+k=l} \mathfrak{t}^{-\mu-j}(z+\mu-k) \mathfrak{s}^{\mu-k}(z) \\
&=\sum_{j+k=l} \sum_{i=1}^{e_{a}}(z-\tilde{p}-l)^{m_{i}^{a}-m_{i}^{a-j-1}}\left(T^{m_{i}^{a-j-1}} \Phi_{i}(\tilde{p})[z-\tilde{p}-l]\right) \\
& \quad \otimes\left(\mathfrak{s}^{\mu-k}(z)^{*} H_{i}^{(a-j-1)}[z-\tilde{p}-l]\right)+O(1) \quad \text { as } z \rightarrow \tilde{p}+l .
\end{aligned}
$$

Since the leading entries of the vectors $T^{m_{i}^{a-j-1}} \Phi_{i}(\tilde{p})$ (if there are any) for different $i$ are linearly independent, we arrive at (4.10).

Step 5. Returning to the notation $H_{i}^{(l)}=H_{i ; a}^{(l)}$, we see that the $\Psi_{i}$ defined by (4.9) for $1 \leq i<e+1$ as $a \rightarrow \infty$ constitute a complete characteristic basis of $L_{\mathfrak{R}^{\mu}}$ modulo $L_{\mathfrak{R}^{\mu}}^{\delta+\mu}$. Furthermore, the considerations also show uniqueness for the complete characteristic basis of $L_{\mathfrak{R}^{\mu}}$ modulo $L_{\mathfrak{R}^{\mu}}^{\delta+\mu}$ just constructed.

\section{Generalization of Keldysh's formula}

Conjugacy of complete characteristic bases in the sense of Theorem 4.1 forces certain bilinear relations between the bases elements to hold, as it does for local asymptotic types. We are now going to derive these relations keeping the notations of the previous section.

Theorem 5.1. For all $p, h, h^{*}, l, j$ satisfying $j \leq l$,

$$
\begin{aligned}
& \sum_{r=j}^{l}\left\langle\boldsymbol{\Theta}_{l-r}\left(T^{m_{h}^{p+l+1}} \Phi_{h}\right)[z+r], T^{m_{h^{*}}^{q+1}} \boldsymbol{I} \Psi_{h^{*}}(q-r)[z-p]\right\rangle \\
& =\delta_{h h^{*}}(z-p)^{-\left(m_{h}^{p}-m_{h}^{p+l+1}\right)}+O\left((z-p)^{-\left(m_{h}^{p}-m_{h}^{p+j}\right)}\right) \quad \text { as } z \rightarrow p,
\end{aligned}
$$

where $q=\operatorname{dim} X-2 \delta-\bar{p}-\mu, m_{h}^{p+j}=m^{p+j}\left(\Phi_{h}\right), m_{h^{*}}^{q+1}=m^{q+1}\left(\Psi_{h^{*}}\right)$,

$$
\delta_{h h^{*}}= \begin{cases}1 & \text { if } h^{*}=\tau^{*}(h), \\ 0 & \text { otherwise, }\end{cases}
$$

and $\boldsymbol{\Theta}_{l-r}\left(T^{m_{h}^{p+l+1}} \Phi_{h}\right)[z+r]$ is defined in (3.9).

Remark 5.2. (a) (5.1) constitutes an asymptotic expansion formula, with $j=l$ being the basic case and further correction terms added as $j$ gets smaller. In Section 7 , we will need the most refined case $j=0$.

(b) In case $l=0$, we recover Keldysh's formula

$$
\begin{aligned}
\left\langle\mathfrak{s}^{\mu}(z) T^{m_{h}^{p+1}} \Phi_{h}(p)[z-p], T^{m_{h^{*}}^{q+1}}\right. & \left.\boldsymbol{I} \Psi_{h^{*}}(q)[z-p]\right\rangle \\
& =\delta_{h h^{*}}(z-p)^{-\left(m_{h}^{p}-m_{h}^{p+1}\right)}+O(1) \quad \text { as } z \rightarrow p .
\end{aligned}
$$

See also (A.2). 
To prove Theorem 5.1 we need the following simple result:

Lemma 5.3. Assume the model situation (4.1). Let $a_{i j}(z) \in \mathcal{A}_{p-l}(\mathbb{C})$ for some $l \in \mathbb{N}_{0}$. Then

$$
\sum_{i=1}^{e_{l}+1} \sum_{j=0}^{l} a_{i j}(z) T^{m_{i}^{j}} \Phi_{i}(p-l)[z-p+l] \in \mathcal{A}_{p-l}\left(C^{\infty}(Y)\right)
$$

if and only if

$$
\sum_{r=0}^{j}(z-p+l)^{m_{i}^{r}} a_{i r}(z)=O\left((z-p+l)^{m_{i}^{j+1}}\right)
$$

for all $1 \leq i \leq e_{l}+1,0 \leq j \leq l$.

Proof of Theorem 5.1. We again assume the model situation (4.1).

We re-enter the scene at formulas (4.8). Using these formulas, we write

$$
\begin{aligned}
\mathfrak{t}^{-\mu-j} & (z+\mu) \\
\quad & =\sum_{b=0}^{a-j-1} \sum_{i=1}^{e_{j+b+1}}\left(T^{m_{i}^{b}} \Phi_{i}(p-j-b)\right) \otimes\left(T^{m_{i}^{a}-m_{i}^{j+b+1}} H_{i}^{(b)}\right)[z-p+b]+G_{j}(z)
\end{aligned}
$$

for $0 \leq j \leq a-1$, where $G_{j}(z)$ is holomorphic on the strip $\operatorname{dim} X / 2-\delta-a+j<$ $\operatorname{Re} z<\operatorname{dim} X / 2-\delta$. For any $0 \leq l^{\prime} \leq a-1$, we get

$$
\begin{aligned}
\delta_{0 l^{\prime}} \text { id }= & \sum_{j+k=l^{\prime}} \mathfrak{t}^{-\mu-j}(z+\mu+j) \mathfrak{s}^{\mu-k}\left(z+l^{\prime}\right) \\
= & \sum_{j+k=l^{\prime}} \sum_{b=0}^{a-l^{\prime}-1} \sum_{i=1}^{e_{l^{\prime}+b+1}}\left(z-p+l^{\prime}+b\right)^{m_{i}^{l^{\prime}+b+1}-m_{i}^{k+b}} \\
& \left(T^{m_{i}^{k+b}} \Phi_{i}\left(p-l^{\prime}-b\right)\left[z-p+l^{\prime}+b\right]\right) \\
& \otimes\left(\mathfrak{s}^{\mu-k}\left(z+l^{\prime}\right)^{*} T^{m_{i}^{a}-m_{i}^{l^{\prime}+b+1}} H_{i}^{(k+b)}\left[z-p+l^{\prime}+b\right]\right) \\
& +\sum_{j+k=l^{\prime}} G_{j}(z+j) \mathfrak{s}^{\mu-k}\left(z+l^{\prime}\right) .
\end{aligned}
$$

We now apply the operator (5.2) to $T^{m_{i^{\prime}}^{\tilde{b}-l}} \Phi_{i^{\prime}}\left(p-\tilde{b}+l^{\prime}\right)[z-p+\tilde{b}]$, where $l \leq \tilde{b} \leq a-1$, and then sum up for $l^{\prime}$ running from 0 to $l$. Since

$$
\begin{aligned}
\sum_{j+k \leq l} & G_{j}(z+j) \mathfrak{s}^{\mu-k}(z+j+k) T^{m_{i^{\prime}}^{\tilde{b}-l}} \Phi_{i^{\prime}}(p-\tilde{b}+j+k)[z-p+\tilde{b}] \\
= & \sum_{j=0}^{l} G_{j}(z+j)\left(\sum_{k=0}^{l-j} \mathfrak{s}^{\mu-k}(z+j+k) T^{m_{i^{\prime}}^{\tilde{b}-l}} \Phi_{i^{\prime}}(p-\tilde{b}+j+k)[z-p+\tilde{b}]\right) \\
= & \sum_{j=0}^{l} G_{j}(z+j) \Theta_{l-j}\left(T^{m_{i^{\prime}}^{\tilde{b}-l}} \Phi_{i^{\prime}}\right)[z+j] \in \mathcal{A}_{p-\tilde{b}}\left(C^{\infty}(Y)\right),
\end{aligned}
$$


taking the principal value at $z=p-\tilde{b}$ on both sides of the resulting equation, we obtain

$$
\begin{aligned}
T^{m_{i^{\prime}}^{\tilde{b}-l}} \Phi_{i^{\prime}}(p-\tilde{b})[z-p+\tilde{b}] & =\sum_{j+k \leq l} \sum_{i=1}^{e_{\tilde{b}+1}}(z-p+\tilde{b})^{m_{i}^{\tilde{b}+1}-m_{i}^{k+b}} \\
\left\langle\mathfrak{s}^{\mu-k}(z+j+k) T^{m_{i^{\prime}}^{\tilde{b}-l}} \Phi_{i^{\prime}}(p-b)[z-p+\tilde{b}], T^{m_{i}^{a}-m_{i}^{\tilde{b}+1}} C H_{i}^{(k+b)}[z-p+\tilde{b}]\right\rangle & T^{m_{i}^{k+b}} \Phi_{i}(p-\tilde{b})[z-p+\tilde{b}]+O(1) \quad \text { as } z \rightarrow p-\tilde{b}
\end{aligned}
$$

where $\tilde{b}=b+j+k$. We get

$$
\begin{aligned}
T^{m_{i^{\prime}}^{\tilde{b}}-l} \Phi_{i^{\prime}}( & p-\tilde{b})[z-p+\tilde{b}]=\sum_{i=1}^{e_{\tilde{b}}+1} \sum_{j=0}^{l} a_{i j}(z) T^{m_{i}^{\tilde{b}-j}} \Phi_{i}(p-\tilde{b})[z-p+\tilde{b}]+O(1) \\
= & \sum_{i=1}^{e_{\tilde{b}}+1} \sum_{j=\tilde{b}-l}^{\tilde{b}} a_{i, \tilde{b}-j}(z) T^{m_{i}^{j}} \Phi_{i}(p-\tilde{b})[z-p+\tilde{b}]+O(1) \quad \text { as } z \rightarrow p-\tilde{b}
\end{aligned}
$$

where

$$
\begin{aligned}
a_{i j}(z)=(z-p+\tilde{b})^{m_{i}^{\tilde{b}+1}-m_{i}^{\tilde{b}-j}} & \\
& \times\left\langle\boldsymbol{\Theta}_{l-j}\left(T^{m_{i^{\prime}}^{\tilde{b}-l}} \Phi_{i^{\prime}}\right)[z+j], T^{m_{i}^{a}-m_{i}^{\tilde{b}+1}} C H_{i}^{(\tilde{b}-j)}[z-p+\tilde{b}]\right\rangle .
\end{aligned}
$$

By virtue of Lemma 5.3, we conclude that, for all $0 \leq j \leq l$,

$$
\begin{aligned}
\sum_{r=j}^{l}(z-p+l)^{m_{i}^{\tilde{b}-r}} & a_{i r}(z) \\
& =\delta_{i i^{\prime}}(z-p+l)^{m_{i}^{\tilde{b}-l}}+O\left((z-p+l)^{m_{i}^{\tilde{b}-j+1}}\right) \quad \text { as } z \rightarrow p-l,
\end{aligned}
$$

i.e.,

$$
\begin{aligned}
& \sum_{r=j}^{l}\left\langle\boldsymbol{\Theta}_{l-r}\left(T^{m_{i^{\prime}}^{\tilde{b}}-l} \Phi_{i^{\prime}}\right)[z+r], T^{m_{i}^{a}-m_{i}^{\tilde{b}+1}} C H_{i}^{(\tilde{b}-r)}[z-p+\tilde{b}]\right\rangle \\
& =\delta_{i i^{\prime}}(z-p+l)^{-\left(m_{i}^{\tilde{b}+1}-m_{i}^{\tilde{\delta}-l}\right)}+O\left((z-p+l)^{-\left(m_{i}^{\tilde{\delta}+1}-m_{i}^{\tilde{b}-j+1}\right)}\right) \quad \text { as } z \rightarrow p-l .
\end{aligned}
$$

In view of (4.9), the latter is (5.1) in the model situation (4.1).

\section{The Boundary SeSQUilinear Form}

In this section, we shall prove Theorem 1.1. From GiL-Mendoza [4, Theorem 7.11], we first quote:

Theorem 6.1. For all $u \in D\left(A_{\max }\right), v \in D\left(A_{\max }^{*}\right)$,

$$
\begin{aligned}
{[u, v]_{A}=} & -\sum_{j=0}^{\mu-1} \sum_{\operatorname{dim} X / 2-\delta-\mu+j<\operatorname{Re} p<\operatorname{dim} X / 2-\delta} \\
& \times \operatorname{Res}_{z=p}\left(\sigma_{c}^{\mu-j}(A)(z)(\omega u)^{\sim}(z),(\omega v)^{\sim}(\operatorname{dim} X-2 \delta-\bar{z}-\mu+j)\right),
\end{aligned}
$$

where $\omega(t)$ is a cut-off function and $(\omega u)^{\sim}(z)=M_{t \rightarrow z}\{(\omega u)(t, \cdot)\}$ denotes Mellin transformation with respect to $t$. 
Proof of Theorem 1.1. We divide the proof into several steps.

Step 1. Since $D\left(A_{\max }\right)=\mathbb{H}_{P_{A}^{\delta}}^{\mu, \delta}(X)$ and $D\left(A_{\min }\right)=\mathbb{H}_{P_{A}^{\delta+\mu-0}}^{\mu, \delta}(X)$ (see Theorem B.6) and similarly for $D\left(A_{\max }^{*}\right), D\left(A_{\text {min }}^{*}\right)$ with $P_{A}^{\delta}$ replaced with $P_{A^{*}}^{\delta}$, we have to compute the induced sesquilinear form

$$
[,]_{A}: L_{\mathfrak{S}^{\mu}}^{\delta} / L_{\mathfrak{S}^{\mu}}^{\delta+\mu-0} \times L_{\mathfrak{R}^{\mu}}^{\delta} / L_{\mathfrak{R}^{\mu}}^{\delta+\mu-0} \rightarrow \mathbb{C} .
$$

Here $\mathfrak{S}^{\mu}=\left\{\mathfrak{s}^{\mu-j}(z) ; j \in \mathbb{N}_{0}\right\}$ and $\mathfrak{R}^{\mu}=\left\{\mathfrak{r}^{\mu-j}(z) ; j \in \mathbb{N}_{0}\right\}$, where $\mathfrak{s}^{\mu-j}(z)=$ $\sigma_{c}^{\mu-j}(A)(z)$ and $\mathfrak{r}^{\mu-j}(z)=\sigma_{c}^{\mu-j}\left(A^{*}\right)(z)$, respectively. For the relation between $\mathfrak{s}^{\mu-j}(z)$ and $\mathfrak{r}^{\mu-j}(z)$, see (3.6). We will evaluate the sesquilinear form (6.2) using formula (6.1).

From (6.2), it is seen that the spaces $D\left(A_{\max }\right) / D\left(A_{\min }\right), D\left(A_{\max }^{*}\right) / D\left(A_{\min }^{*}\right)$ are invariant under the action of the operator $T$ from (1.6). (This result is implicitly contained in Theorem 2.12)

Step 2. It suffices to prove (1.9) for an arbitrary characteristic basis $\Phi_{1}, \ldots, \Phi_{e}$ of the quotient $L_{\mathfrak{S}^{\mu}}^{\delta} / L_{\mathfrak{S}^{\mu}}^{\delta+\mu-0}$; see again Theorem B.6. For then non-degeneracy of the sesquilinear form (6.2) and also property (1.10) follow, where the latter holds for all $\Phi \in L_{\mathfrak{S}^{\mu}}^{\delta} / L_{\mathfrak{S}^{\mu}}^{\delta+\mu-0}, \Psi \in L_{\mathfrak{R}^{\mu}}^{\delta} / L_{\mathfrak{R}^{\mu}}^{\delta+\mu-0}$. Moreover, if $\Phi_{1}^{\prime}, \ldots, \Phi_{e}^{\prime}$ is another characteristic basis of the quotient $L_{\mathfrak{S}^{\mu}}^{\delta} / L_{\mathfrak{S}^{\mu}}^{\delta+\mu-0}$, then we have (after renumbering if necessary)

$$
\Phi_{i}^{\prime}=C \Phi_{i}, \quad 1 \leq i \leq e,
$$

for some linear invertible operator $C: L_{\mathfrak{S}^{\mu}}^{\delta} / L_{\mathfrak{S}^{\mu}}^{\delta+\mu-0} \rightarrow L_{\mathfrak{S}^{\mu}}^{\delta} / L_{\mathfrak{S}^{\mu}}^{\delta+\mu-0}$ that commutes with $T$. Denoting by $C^{*}$ the adjoint to $C$ with respect to the non-degenerate sesquilinear form (6.2) $\left(C^{*}\right.$ also commutes with $\left.T\right)$, the conjugate characteristic basis $\Psi_{1}^{\prime}, \ldots, \Psi_{e}^{\prime}$ to $\Phi_{1}^{\prime}, \ldots, \Phi_{e}^{\prime}$ is given by

$$
\Psi_{i}^{\prime}=\left(C^{*}\right)^{-1} \Psi_{i}, \quad 1 \leq i \leq e,
$$

where $\Psi_{1}, \ldots, \Psi_{e}$ is the conjugate characteristic basis to $\Phi_{1}, \ldots, \Phi_{e}$, and (1.9) follows for $\Phi_{1}^{\prime}, \ldots, \Phi_{e}^{\prime}$, too.

Step 3. Let $\Phi_{1}, \ldots, \Phi_{e}$ be a characteristic basis of $L_{\mathfrak{S}^{\mu}}^{\delta} / L_{\mathfrak{S}^{\mu}}^{\delta+\mu-0}$, of characteristic $\left(m_{1}, \ldots, m_{e}\right)$ say, and let $\Psi_{1}, \ldots, \Psi_{e}$ be the conjugate characteristic basis of $L_{\mathfrak{R}^{\mu}}^{\delta} / L_{\mathfrak{R}^{\mu}}^{\delta+\mu-0}$ according to Theorem 4.1] meaning that there are corresponding versions of Theorems 4.1, 5.1 valid for the Mellin symbols $\mathfrak{t}^{-\mu-j}(z)$ for $0 \leq j<\mu$ in the strip $\operatorname{dim} X / 2-\delta-\mu+j<\operatorname{Re} z<\operatorname{dim} X / 2-\delta-\mu$, where now in (4.2), (5.1) elements of the quotients $L_{\mathfrak{S}^{\mu}}^{\delta} / L_{\mathfrak{S}^{\mu}}^{\delta+\mu-0}, L_{\mathfrak{R}^{\mu}}^{\delta} / L_{\mathfrak{R}^{\mu}}^{\delta+\mu-0}$ enter. Likewise, we may assume that $\Phi_{1}, \ldots, \Phi_{e}$ stem (by projection) from a characteristic basis of $L_{\mathfrak{S}^{\mu}}^{\delta}$ that can be extended to a complete characteristic basis of $L_{\mathfrak{S}^{\mu}}$.

We will make this latter assumption to keep the notation from Theorems 4.1 . 5.1

For $\Phi \in L_{\mathfrak{S}^{\mu}}^{\delta} / L_{\mathfrak{S}^{\mu}}^{\delta+\mu-0}, \Psi \in L_{\mathfrak{R}^{\mu}}^{\delta} / L_{\mathfrak{R}^{\mu}}^{\delta+\mu-0}$, we rewrite (6.1) as

$$
\begin{aligned}
& {[\Phi, \Psi]_{A}=-\sum_{k=0}^{\mu-1} \sum_{\operatorname{dim} X / 2-\delta-\mu+k<\operatorname{Re} p<\operatorname{dim} X / 2-\delta}} \\
& \times \operatorname{Res}_{z=p}\left\langle\mathfrak{s}^{\mu-k}(z) \Phi(p)[z-p], \boldsymbol{I} \Psi(q+k)[z-p]\right\rangle,
\end{aligned}
$$

where $q=\operatorname{dim} X-2 \delta-\bar{p}-\mu$.

Now choose $\Phi$ belonging to the Jordan basis $\Phi_{1}, \ldots, T^{m_{1}-1} \Phi_{1}, \ldots, \Phi_{e}, \ldots$, $T^{m_{e}-1} \Phi_{e}$ of the quotient $L_{\mathfrak{S}^{\mu}}^{\delta} / L_{\mathfrak{S}^{\mu}}^{\delta+\mu-0}$ and $\Psi$ belonging to the conjugate Jordan 
basis $\Psi_{1}, \ldots, T^{m_{1}-1} \Psi_{1}, \ldots, \Psi_{e}, \ldots, T^{m_{e}-1} \Psi_{e}$ of the quotient $L_{\mathfrak{R}^{\mu}}^{\delta} / L_{\mathfrak{R}^{\mu}}^{\delta+\mu-0}$. That means that

$$
\Phi=T^{i} T_{h}^{m_{h}^{p+l+1}} \Phi_{h}
$$

for some $h, p, l, i$, where $\operatorname{dim} X / 2-\delta-\mu<\operatorname{Re} p \leq \operatorname{dim} X / 2-\delta-(\mu-1), \operatorname{Re} p+l \leq$ $\operatorname{dim} X / 2-\delta$, and $0 \leq i<m_{h}^{p+l}-m_{h}^{p+l+1}$. We may further assume that

$$
\Psi=T^{j} T^{m_{h^{*}}^{q+1}} \Psi_{h^{*}},
$$

where $q=\operatorname{dim} X-2 \delta-\bar{p}-\mu$ and $0 \leq j<m_{h^{*}}^{q+l+1}-m_{h^{*}}^{q+1}$, since otherwise $[\Phi, \Psi]_{A}=0$.

Under these hypotheses, in (6.3) there are non-zero residues at most at $z=p+r$ for $r=0, \ldots, l$, i.e.,

$$
\begin{aligned}
& {[\Phi, \Psi]_{A}=(-1)^{j+1} \sum_{k=0}^{l} \sum_{r=k}^{l} \operatorname{Res}_{z=p+r}\left\langle\mathfrak{s}^{\mu-k}(z) T^{i} T^{m_{h}^{p+l+1}} \Phi_{h}(p+r)[z-p-r],\right.} \\
& \left.T^{j} \boldsymbol{I} T^{m_{h^{*}}^{q+1}} \Psi_{h^{*}}(q-r+k)[z-p-r]\right\rangle \\
& =(-1)^{j+1} \sum_{k=0}^{l} \sum_{r=k}^{l} \operatorname{Res}_{z=p+r-k} \\
& \left\langle\mathfrak{s}^{\mu-k}(z+k) T^{i} T^{m_{h}^{p+l+1}} \Phi_{h}(p+r)[z-p-r+k],\right. \\
& \left.T^{j} \boldsymbol{I} T^{m_{h^{*}}^{q+1}} \Psi_{h^{*}}(q-r+k)[z-p-r+k]\right\rangle \\
& =(-1)^{j+1} \sum_{k=0}^{l} \sum_{r=0}^{l-k} \operatorname{Res}_{z=p+r} \\
& \left\langle\mathfrak{s}^{\mu-k}(z+k) T^{i} T^{m_{h}^{p+l+1}} \Phi_{h}(p+r+k)[z-p-r],\right. \\
& \left.T^{j} \boldsymbol{I} T^{m_{h^{*}}^{q+1}} \Psi_{h^{*}}(q-r)[z-p-r]\right\rangle \\
& =(-1)^{j+1} \sum_{r=0}^{l} \operatorname{Res}_{z=p+r} \\
& \left\langle\sum_{k=0}^{l-r} \mathfrak{s}^{\mu-k}(z+k) T^{i} T_{h}^{m_{h}^{p+l+1}} \Phi_{h}(p+r+k)[z-p-r],\right. \\
& \left.T^{j} \boldsymbol{I} T^{m_{h^{*}}^{q+1}} \Psi_{h^{*}}(q-r)[z-p-r]\right\rangle \\
& =(-1)^{j+1} \sum_{r=0}^{l} \operatorname{Res}_{z=p+r}(z-p-r)^{i+j}\left\langle\boldsymbol{\Theta}_{l-r}\left(T^{m_{h}^{p+l+1}} \Phi_{h}\right)[z],\right. \\
& \left.\boldsymbol{I} T^{m_{h^{*}}^{q+1}} \Psi_{h^{*}}(q-r)[z-p-r]\right\rangle \\
& =(-1)^{j+1} \operatorname{Res}_{z=p}(z-p)^{i+j} \sum_{r=0}^{l}\left\langle\boldsymbol{\Theta}_{l-r}\left(T^{m_{h}^{p+l+1}} \Phi_{h}\right)[z+r],\right. \\
& \left.\boldsymbol{I} T^{m_{h^{*}}^{q+1}} \Psi_{h^{*}}(q-r)[z-p]\right\rangle .
\end{aligned}
$$


Therefore,

$$
[\Phi, \Psi]_{A}= \begin{cases}(-1)^{j+1} & \text { if } \tau^{*}(h)=h^{*}, i+j=m_{h}^{p}-m_{h}^{p+l+1}-1, \\ 0 & \text { otherwise }\end{cases}
$$

by virtue of Theorem 5.1

The proof is complete.

\section{EXAMPLES}

We discuss two examples of ordinary differential operators on the half-line $\mathbb{R}_{+}$. The first example demonstrates the usage of Theorem 1.1 for the computation of the boundary sesquilinear form, while in the second example it is shown how our fundamental formulas like (4.2) can be independently verified.

7.1. First example. This example concerns the cone-degenerate third-order operator

$$
A=\partial_{t}^{3}+t^{-1} \partial_{t}^{2} \quad \text { on } \mathbb{R}_{+} .
$$

The conormal symbols are

$$
\sigma_{c}^{3}(A)(z)=-z(z+1)^{2}
$$

and $\sigma_{c}^{3-j}(A)(z)=0$ for $j \geq 1$. Thus, $1, t \log t$, and $t$ are exact solutions to the equation $A u=0$. A complete characteristic basis $\Phi_{1}, \Phi_{2}$ of $L_{A}$ is given by

$$
\Phi_{1}(0)=(1), \quad \Phi_{2}(-1)=(1,0),
$$

and $\Phi_{1}(p)=0$ for $p \neq 0, \Phi_{2}(p)=0$ for $p \neq-1$.

We choose $\boldsymbol{\delta}=\mathbf{- 1}$. Then we have $A^{*}=-\partial_{t}^{3}-5 t^{-1} \partial_{t}^{2}-4 t^{-2} \partial_{t}, \sigma_{c}^{3}\left(A^{*}\right)(z)=$ $z(z-1)^{2}$, and $\sigma_{c}^{3-j}\left(A^{*}\right)(z)=0$ for $j \geq 1$. From

$$
-\frac{1}{z(z+1)^{2}}=\frac{1}{(z+1)^{2}}+\frac{1}{z+1}-\frac{1}{z}
$$

in conjunction with formula (4.2) we infer that the complete characteristic basis $\Psi_{1}, \Psi_{2}$ of $L_{A^{*}}$ that is conjugate to $\Phi_{1}, \Phi_{2}$ is given by

$$
\Psi_{1}(1)=(1,-1), \quad \Psi_{2}(0)=(1),
$$

and $\Psi_{1}(p)=0$ for $p \neq 1, \Psi_{2}(p)=0$ for $p \neq 0$, where $\tau^{*}(1)=2, \tau^{*}(2)=1$.

Writing

$$
\begin{aligned}
& u(t)=\omega(t)\left(\alpha+\beta_{0} t \log t+\beta_{1} t\right)+u_{0}(t), \\
& v(t)=\omega(t)\left(\gamma_{0} t^{-1} \log t+\gamma_{1} t^{-1}+\delta\right)+v_{0}(t)
\end{aligned}
$$

for $\alpha, \beta_{0}, \beta_{1}, \gamma_{0}, \gamma_{1}, \delta \in \mathbb{C}$, where $\omega(t)$ is a cut-off function and $u_{0} \in D\left(A_{\min }\right)$, $v_{0} \in D\left(A_{\mathrm{min}}^{*}\right)$, we then obtain

$$
[u, v]_{A}=-\alpha \bar{\delta}+\beta_{0} \bar{\gamma}_{0}+\beta_{0} \bar{\gamma}_{1}-\beta_{1} \bar{\gamma}_{0}
$$

in view of formula (1.9). 
7.2. Second example. We consider the non-degenerate, second-order, constant coefficient operator

$$
A=\partial_{t}^{2}+a \partial_{t}+b \quad \text { on } \mathbb{R}_{+},
$$

where $a, b \in \mathbb{C}$. We have $A^{*}=\partial_{t}^{2}-\bar{a} \partial_{t}+\bar{b}$ (with $\boldsymbol{\delta}=\mathbf{0}$ ) and Green's formula is directly checked to be

$$
[u, v]_{A}=u(0) \bar{v}^{\prime}(0)-u^{\prime}(0) \bar{v}(0)-a u(0) \bar{v}(0), \quad u, v \in \mathcal{S}\left(\overline{\mathbb{R}}_{+}\right) .
$$

The space $L_{A}^{0}$ has characteristic $\{(-j ; 1,1, \ldots) ; j=0,1\}$. Therefore, $L_{A}^{2-0}=$ $\{0\}$, the quotient $D\left(A_{\max }\right) / D\left(A_{\min }\right) \cong L_{A}^{0}$ is two-dimensional, and the elements of $D\left(A_{\max }\right) / D\left(A_{\min }\right)$ are in one-to-one correspondence with the (in fact, analytic) solutions $u(t)=u(t ; \alpha, \beta)$ for $\alpha, \beta \in \mathbb{C}$ to

$$
A u=0, \quad u(0)=\alpha, \quad u^{\prime}(0)=\beta .
$$

In the following, we shall assume this identification to be made.

A complete characteristic basis of $L_{A}=L_{A}^{0}$ is given by

$$
u_{1}(t)=u(t ; 1,0), \quad u_{2}(t)=u(t ; 0,1) .
$$

We look at (7.1) to find the conjugate complete characteristic basis of $L_{A^{*}}$ to be

$$
v_{1}(t)=u(t ; 1, \bar{a}), \quad v_{2}(t)=v(t ; 0,-1),
$$

where $v(t)=v(t ; \alpha, \beta)$ is the solution to $A^{*} v=0, v(0)=\alpha, v^{\prime}(0)=\beta$.

Proposition 7.1. (a) We have

$$
u_{1}(t)=1+\sum_{j \geq 2}(-1)^{j-1} \frac{b \Pi_{j-2}(a, b)}{j !} t^{j}, \quad u_{2}(t)=\sum_{j \geq 1}(-1)^{j-1} \frac{\Pi_{j-1}(a, b)}{j !} t^{j}
$$

and

$$
v_{1}(t)=\sum_{j \geq 0} \frac{\Pi_{j}(\bar{a}, \bar{b})}{j !} t^{j}, \quad v_{2}(t)=-\sum_{j \geq 1} \frac{\Pi_{j-1}(\bar{a}, \bar{b})}{j !} t^{j},
$$

where $\Pi_{0}(a, b)=1, \Pi_{1}(a, b)=a$,

$$
\Pi_{j}(a, b)=a \Pi_{j-1}(a, b)-b \Pi_{j-2}(a, b), \quad j=2,3, \ldots
$$

(i.e., $\Pi_{2}(a, b)=a^{2}-b, \Pi_{3}(a, b)=a^{3}-2 a b, \Pi_{4}(a, b)=a^{4}-3 a^{2} b+b^{2}$, etc.).

(b) We also have

$$
\mathfrak{t}^{-k-2}(z+2)=\frac{\Pi_{k}(a, b)}{(z-k)(z-k+1) \ldots z(z+1)}, \quad k=0,1,2, \ldots,
$$

where $\mathfrak{t}^{-k-2}(z)$ has the same meaning as before. In particular, the poles of $\mathfrak{t}^{-k-2}(z)$ are simple and, for $l=-1,0,1, \ldots, k$,

$$
\operatorname{Res}_{z=l} \mathfrak{t}^{-k-2}(z+2)=\frac{(-1)^{k-l} \Pi_{k}(a, b)}{(k-l) !(l+1) !} .
$$

The key in re-proving formulas like (4.2) is the following elementary result:

Lemma 7.2. (i) For $l \geq 2,0 \leq j \leq l-2$,

$$
\Pi_{l}(a, b)=\Pi_{j+1}(a, b) \Pi_{l-j-1}(a, b)-b \Pi_{j}(a, b) \Pi_{l-j-2}(a, b) .
$$

(ii) For $j \geq 0$,

$$
\Pi_{j}(-\bar{a}, \bar{b})=(-1)^{j} \overline{\Pi_{j}(a, b)} .
$$




\section{Appendix A. Local ASYMPtotic types}

We briefly discuss the notion of local asymptotic type, i.e., asymptotic types at one singular exponent $p \in \mathbb{C}$ in (1.5). Moreover, we investigate an analogue of the boundary sesquilinear form in this simpler situation; see (A.3). Most of the material is taken from WiтT [21].

Let $E$ be a Banach space, let $E^{\prime}$ be its topological dual, and let $\langle$,$\rangle denote the$ dual pairing between $E, E^{\prime}$. Pick $p \in \mathbb{C}$. For notations like $\mathcal{M}_{p}(E), \mathcal{A}_{p}(E), E^{\infty}$, the right-shift operator $T$ acting on $E^{\infty}, \Phi \otimes \Psi[z-p]$ for $\Phi \in E^{\infty}, \Psi \in E^{\prime \infty}$, and the identification $\mathcal{M}_{p}(E) / \mathcal{A}_{p}(E) \cong E^{\infty}$, see Section 1.3 .

Let $\mathcal{M}_{p}^{\text {fin }}(\mathcal{L}(E))$ be the space of germs of $\mathcal{L}(E)$-valued finitely meromorphic functions $F(z)$ at $z=p$, i.e.,

$$
F(z)=\frac{F_{0}}{(z-p)^{\nu}}+\frac{F_{1}}{(z-p)^{\nu-1}}+\cdots+\frac{F_{\nu-1}}{z-p}+\sum_{j \geq 0} F_{j}(z-p)^{j}
$$

where $F_{0}, F_{1}, \ldots, F_{\nu-1} \in \mathcal{L}(E)$ are finite-rank operators. Let $\mathcal{M}_{p}^{\text {nor }}(\mathcal{L}(E))$ be the space of germs of $\mathcal{L}(E)$-valued normally meromorphic functions $F(z)$ at $z=p$, i.e., the space of finitely meromorphic functions $F(z)$, where, in addition, $F(z)$ for $z \neq p$ close to $p$ is invertible and $F_{\nu} \in \mathcal{L}(E)$ is a Fredholm operator (then necessarily of index 0). Note that $\mathcal{M}_{p}^{\text {nor }}(\mathcal{L}(E))$ is the group of invertible elements of the algebra $\mathcal{M}_{p}^{\text {fin }}(\mathcal{L}(E))$.

For $F \in \mathcal{A}_{p}(\mathcal{L}(E))$, let $L_{F}$ denote the space of all $\left(\phi_{0}, \phi_{1}, \ldots, \phi_{m-1}\right) \in E^{\infty}$ such that

$$
F(z)\left(\frac{\phi_{0}}{(z-p)^{m}}+\frac{\phi_{1}}{(z-p)^{m-1}}+\cdots+\frac{\phi_{m-1}}{(z-p)}\right) \in \mathcal{A}_{p}(E) .
$$

Remark A.1. The theory can also be developed for $F \in \mathcal{M}_{p}^{\text {fin }}(\mathcal{L}(E))$ upon an appropriate modification of the definition of $L_{F}$. For $F \in \mathcal{M}_{p}^{\text {nor }}(\mathcal{L}(E)), L_{F}$ is again an asymptotic type, and Propositions A.4, A.5, and A.7 continue to hold in this case. See Witt [21].

Definition A.2. A local asymptotic type $J \subset E^{\infty}$ is a finite-dimensional linear subspace that is invariant under the action of the right shift operator $T$. The set of all local asymptotic types is denoted by $\mathcal{J}(E)$.

Note that $T$ as acting on $J$ is nilpotent. The characteristic $\left(m_{1}, \ldots, m_{e}\right)$ of $T$ on $J$ is called the characteristic of the asymptotic type.

Proposition A.3. We have

$$
\mathcal{J}(E)=\left\{L_{F} \mid F \in \mathcal{A}_{p}(\mathcal{L}(E)) \cap \mathcal{M}_{p}^{\text {nor }}(\mathcal{L}(E))\right\} .
$$

Proposition A.4. For $F \in \mathcal{A}_{p}(\mathcal{L}(E)) \cap \mathcal{M}_{p}^{\text {nor }}(\mathcal{L}(E))$, we have $F^{t} \in \mathcal{A}_{p}\left(\mathcal{L}\left(E^{\prime}\right)\right) \cap$ $\mathcal{M}_{p}^{\text {nor }}\left(\mathcal{L}\left(E^{\prime}\right)\right)$. Moreover, for each characteristic basis $\Phi_{1}, \ldots, \Phi_{e}$ of $L_{F}$, there exists a unique characteristic basis $\Psi_{1}, \ldots, \Psi_{e}$ of $L_{F^{t}}$ such that

$$
\left[F^{-1}(z)\right]_{p}^{*}=\sum_{i=1}^{e}\left(\Phi_{i} \otimes \Psi_{i}\right)[z-p] .
$$

In particular, both asymptotic types $L_{F}, L_{F^{t}}$ have the same characteristic. 
The next result is Keldysh's formula; see Keldysh [7, Kozlov-Maz'ya [9].

Proposition A.5. For $\Phi_{1}, \ldots, \Phi_{e}$ and $\Psi_{1}, \ldots, \Psi_{e}$ as in Proposition A.4.

$$
\left\langle F(z) \Phi_{i}[z-p], \Psi_{j}[z-p]\right\rangle=\delta_{i j}(z-p)^{-m_{i}}+O(1) \quad \text { as } z \rightarrow p .
$$

Remark A.6. Writing $F(z)$ as in (A.1), (A.2) can be rewritten as

$$
\sum_{\nu+r+s=m_{i}+l}\left\langle F_{\nu} \phi_{r}^{(i)}, \psi_{s}^{(j)}\right\rangle=\delta_{i j} \delta_{0 l}
$$

for $0 \leq l \leq m_{j}-1$, where summation is restricted to the range $0 \leq r \leq m_{i}-1$, $0 \leq s \leq m_{j}-1$, and $\Phi_{i}=\left(\phi_{0}^{(i)}, \phi_{1}^{(i)}, \ldots, \phi_{m_{i}-1}^{(i)}\right), \Psi_{j}=\left(\psi_{0}^{(j)}, \psi_{1}^{(j)}, \ldots, \psi_{m_{j}-1}^{(j)}\right)$.

For $F \in \mathcal{A}_{p}(\mathcal{L}(E)) \cap \mathcal{M}_{p}^{\text {nor }}(\mathcal{L}(E))$, we finally introduce the bilinear form $[,]_{F}$ defined on the product $L_{F} \times L_{F^{t}}$ by

$$
[\Phi, \Psi]_{F}:=\operatorname{Res}_{z=p}\langle F(z) \Phi[z-p], \Psi[z-p]\rangle .
$$

Proposition A.7. Evaluated on the bases $T^{r} \Phi_{i}$ for $1 \leq i \leq e, 0 \leq r \leq m_{i}-1$ of $L_{F}$ and $T^{s} \Psi_{j}$ for $1 \leq j \leq e, 0 \leq s \leq m_{j}-1$ of $L_{F^{t}}$,

$$
\left[T^{r} \Phi_{i}, T^{s} \Psi_{j}\right]_{F}= \begin{cases}1 & \text { if } i=j, r+s=m_{i}-1, \\ 0 & \text { otherwise. }\end{cases}
$$

Proof. This follows immediately from Proposition A.5

\section{Appendix B. Function spaces With asymptotics}

The maximal and minimal domains of elliptic cone differential operators are cone Sobolev spaces with asymptotics, as we are going to demonstrate now. We refer to SCHulze [16, 17] for more on function spaces with asymptotics, where, however, asymptotics are observed on so-called "half-open weight intervals," a setting leading to Fréchet spaces. The present setting due to LiU-WiTT [12, where asymptotics are observed on "closed weight intervals," provides a scale of Hilbert spaces.

B.1. Weighted cone Sobolev spaces. Let $M u(z)=\tilde{u}(z)=\int_{0}^{\infty} t^{z-1} u(t) d t$ for $z \in \mathbb{C}$ (or subsets thereof) be the Mellin transformation, suitably extended to certain distribution classes. Recall that

$$
M: L^{2}\left(\mathbb{R}_{+}, t^{-2 \delta} d t\right) \rightarrow L^{2}\left(\Gamma_{1 / 2-\delta} ;(2 \pi i)^{-1} d z\right)
$$

is an isometry, where $\Gamma_{\gamma}:=\{z \in \mathbb{C} \mid \operatorname{Re} z=\gamma\}$ for $\gamma \in \mathbb{R}$. Moreover,

$$
\begin{aligned}
& M_{t \rightarrow z}\left\{\left(-t \partial_{t}\right) u\right\}(z)=z \tilde{u}(z), \\
& M_{t \rightarrow z}\left\{t^{-p} u\right\}(z)=\tilde{u}(z-p), \quad p \in \mathbb{C} .
\end{aligned}
$$

The function

$$
\mathfrak{m}_{p, k}(z, y):=M_{t \rightarrow z}\left\{\frac{(-1)^{k}}{k !} \omega(t) t^{-p} \log ^{k} t \phi(y)\right\},
$$

where $p \in \mathbb{C}, k \in \mathbb{N}_{0}, \phi \in C^{\infty}(Y)$, and $\omega(t)$ is a cut-off function, belongs to $\mathcal{M}_{\mathrm{as}}^{-\infty}(Y)$. Furthermore,

$$
\mathfrak{m}_{p, k}(z)-\frac{\phi(y)}{(z-p)^{k+1}} \in \mathcal{A}\left(\mathbb{C} ; C^{\infty}(Y)\right) .
$$


For $s, \delta \in \mathbb{R}$, the Hilbert space $\mathcal{H}^{s, \delta}(X)$ consists of all $u \in H_{\mathrm{loc}}^{s}\left(X^{\circ}\right)$ such that $M_{t \rightarrow z}\{\omega u\}(z) \in L_{\mathrm{loc}}^{2}\left(\Gamma_{\operatorname{dim} X / 2-\delta} ; H^{s}(Y)\right)$ and

$$
\frac{1}{2 \pi i} \int_{\Gamma_{\operatorname{dim} X / 2-\delta}}\left\|R^{s}(z) M_{t \rightarrow z}\{\omega u\}(z)\right\|_{L^{2}(Y)}^{2} d z<\infty .
$$

Here, $R^{s}(z) \in L^{s}\left(Y ; \Gamma_{\operatorname{dim} X / 2-\delta}\right)$ is an order-reducing family, i.e., $R^{s}(z)$ is parameter-dependent elliptic and $R^{s}(z): H^{s+s^{\prime}}(Y) \rightarrow H^{s^{\prime}}(Y)$ is invertible for all $s^{\prime} \in \mathbb{R}$, $z \in \Gamma_{\operatorname{dim} X / 2-\delta}$. For instance, if $\mathfrak{m}(z) \in \mathcal{M}_{\text {as }}^{s}(Y)$ is elliptic and the line $\Gamma_{\operatorname{dim} X / 2-\delta}$ is free of poles of $\mathfrak{m}(z)$, then $\left.\mathfrak{m}(z)\right|_{\Gamma_{\operatorname{dim} X / 2-\delta}}$ is such an order-reduction.

B.2. Cone Sobolev spaces with asymptotics. The starting point is the following observation:

Theorem B.1 (LiU-WitT [12, Theorem 2.43]). Let $s, \delta \in \mathbb{R}$ and $P \in \underline{\mathrm{As}^{\delta}}(Y)$ be a proper asymptotic type. Then there exists an elliptic Mellin symbol $\mathfrak{m}_{P}^{s}(z) \in$ $\mathcal{M}_{\mathcal{O}}^{s}(Y)$ such that the line $\Gamma_{\operatorname{dim} X / 2-\delta}$ is free of poles of $\mathfrak{m}_{P}^{s}(z)^{-1}$ and, for $\mathfrak{S}^{s}=$ $\left\{\mathfrak{m}_{P}^{s}(z), 0,0, \ldots\right\} \in \operatorname{Symb}_{M}^{s}(Y), L_{\mathfrak{S}^{s}}^{\delta}$ represents the asymptotic type $P$.

Definition B.2. Let $s \geq 0, \delta \in \mathbb{R}$, and $P \in \underline{A s}^{\delta}(Y)$ be proper. Then the space $\mathbb{H}_{P}^{s, \delta}(X)$ consists of all functions $u \in \mathcal{H}^{s, \delta}(X)$ such that $M_{t \rightarrow z}\{\omega u\}(z)$ is meromorphic for $\operatorname{Re} z>\operatorname{dim} X / 2-\delta-s$ with values in $H^{s}(Y)$,

$$
\mathfrak{m}_{P}^{s}(z) M_{t \rightarrow z}\{\omega u\}(z) \in \mathcal{A}\left(\{z \in \mathbb{C} \mid \operatorname{Re} z>\operatorname{dim} X / 2-\delta-s\} ; L^{2}(Y)\right),
$$

where $\mathfrak{m}_{P}^{s}(z)$ is as in Theorem B.1, and

$$
\sup _{0<s^{\prime}<s} \frac{1}{2 \pi i} \int_{\Gamma_{\mathrm{dim} X / 2-\delta-s^{\prime}}}\left\|\mathfrak{m}_{P}^{s}(z) M_{t \rightarrow z}\{\omega u\}(z)\right\|_{L^{2}(Y)}^{2} d z<\infty .
$$

We list some properties of the spaces $\mathbb{H}_{P}^{s, \delta}(X)$ :

Proposition B.3. (a) $\left\{\mathbb{H}_{P}^{s, \delta}(X) ; s \geq 0\right\}$ is an interpolation scale of Hilbert spaces with respect to the complex interpolation method.

(b) $\mathbb{H}_{\mathcal{O}}^{s, \delta}(X)=\mathcal{H}^{s, \delta+s}(X)$.

(c) We have

$$
\begin{array}{r}
\mathbb{H}_{P}^{s, \delta}(X)=\mathbb{H}_{\mathcal{O}}^{s, \delta}(X) \oplus\left\{\omega(t) \sum_{\operatorname{Re} p>\operatorname{dim} X / 2-\delta-s} \sum_{k+l=m_{p}-1} \frac{(-1)^{k}}{k !} t^{-p} \log ^{k} t \phi_{l}^{(p)}(y) \mid\right. \\
\left.\Phi(p)=\left(\phi_{0}^{(p)}, \ldots, \phi_{m_{p}-1}^{(p)}\right) \text { for some } \Phi \in J\right\}
\end{array}
$$

where the linear space $J \subset \mathcal{E}_{V}^{\delta}(Y)$ represents the asymptotic type $P$, provided that

$$
\operatorname{Re} p \neq \operatorname{dim} X / 2-\delta-s, \quad p \in V .
$$

(d) $\mathbb{H}_{P}^{s, \delta}(X) \subseteq \mathbb{H}_{P^{\prime}{ }^{\prime}, \delta^{\prime}}(X)$ if and only if $s \geq s^{\prime}, \delta+s \geq \delta^{\prime}+s^{\prime}$, and $P \preccurlyeq P^{\prime}$ up to the conormal order $\delta^{\prime}+s^{\prime}$.

(e) $C_{P}^{\infty}(X):=\bigcap_{s \geq 0} \mathbb{H}_{P}^{s, \delta}(X)$ is dense in $\mathbb{H}_{P}^{s, \delta}(X)$.

Proposition B.4. The spaces $\mathbb{H}_{P}^{s, \delta}(X)$ are invariant under coordinate changes in the sense explained in Remark 2.5 . 
B.3. Mapping properties and elliptic regularity. Here we are concerned with the regularity and asymptotics of solutions $u$ to the equation

$$
A u(x)=f(x) \quad \text { on } X^{\circ},
$$

where $A \in \operatorname{Diff}_{\text {cone }}^{\mu}(X)$ is elliptic. Assuming $u \in \mathbb{H}^{0, \delta}(X)$ and $f \in \mathbb{H}_{Q}^{s, \delta}(X)$, where $s \geq 0$ and $Q \in \underline{\mathrm{As}^{\delta}}(Y)$, we are going to show that $u \in \mathbb{H}_{P}^{s+\mu, \delta}(X)$ for some resulting $P \in \underline{\mathrm{As}^{\delta}}(Y)$. By interior elliptic regularity, we already know that $u \in H_{\mathrm{loc}}^{s+\mu}\left(X^{\circ}\right)$. So we are left with the behavior of $u=u(x)$ as $x \rightarrow \partial X$.

Let $P_{A}^{\delta}$ be the asymptotic type represented by $L_{A}^{\delta}$; see Definition 3.8(b). Similarly, let $P_{A}^{\delta+\mu-0} \preccurlyeq P_{A}^{\delta}$ be the asymptotic type represented by $L_{A}^{\delta+\mu-0}$. Then $P_{A}^{\delta+\mu-0}$ is the largest asymptotic type that coincides with the empty asymptotic type, $\mathcal{O}$, up to the conormal order $\delta+\mu-0$. Note that, for each $P \in \underline{\mathrm{As}}{ }^{\delta}(Y)$ satisfying $P \preccurlyeq P_{A}^{\delta}$ up to the conormal order $\delta+\mu$, there is a $Q \in \underline{A s} \delta(Y)$ such that

$$
A: \mathbb{H}_{P}^{s+\mu, \delta}(X) \rightarrow \mathbb{H}_{Q}^{s, \delta}(X)
$$

for all $s \geq 0$. The minimal such $Q \in \underline{\mathrm{As}}^{\delta}(Y)$ is denoted by $Q^{\delta}(P ; A)$. In particular, $Q^{\delta}\left(P_{A} ; A\right)=Q^{\delta}(\mathcal{O} ; A)=\mathcal{O}$.

The question raised for equation (B.1) is answered by the next result:

Proposition B.5. Let $A \in \operatorname{Diff}_{\text {cone }}^{\mu}(X)$ be elliptic. Then:

(a) The map

$$
\begin{aligned}
\left\{P \in \underline{\mathrm{As}}^{\delta}(Y): P\right. & \succcurlyeq P_{A}^{\delta}, P \text { coincides with } P_{A}^{\delta} \text { up to } \\
& \text { the conormal order } \delta+\mu\} \rightarrow \underline{\mathrm{As}^{\delta}}(Y), \quad P \mapsto Q^{\delta}(P ; A)
\end{aligned}
$$

is an order-preserving bijection.

(b) For any solution $u$ to (B.1), $u \in \mathbb{H}^{0, \delta}(X)$ and $f \in \mathbb{H}_{Q}^{s, \delta}(X)$ implies $u \in$ $\mathbb{H}_{P^{\delta}(Q ; A)}^{s+\mu, \delta}(X)$, where $Q \mapsto P^{\delta}(Q ; A)$ is the inverse to (B.2).

Note that $Q^{\delta}\left(P^{\delta}(Q ; A) ; A\right)=Q$. Therefore, $P \mapsto P^{\delta}\left(Q^{\delta}(P ; A) ; A\right)$ is a hull operation. Note also that both maps $P \rightarrow Q^{\delta}(P ; A)$ and $Q \mapsto P^{\delta}(Q ; A)$ to (B.2) can be computed purely on the level of the complete conormal symbols $\left\{\sigma_{c}^{\mu-j}(A)(z) ; j \in\right.$ $\left.\mathbb{N}_{0}\right\}$.

Theorem B.6. Let $A \in \operatorname{Diff}_{\text {cone }}^{\mu}(X)$ be elliptic. Then

$$
D\left(A_{\max }\right)=\mathbb{H}_{P_{A}^{\delta}}^{\mu, \delta}(X), \quad D\left(A_{\min }\right)=\mathbb{H}_{P_{A}^{\delta+\mu-0}}^{\mu, \delta}(X) .
$$

In particular,

$$
D\left(A_{\max }\right) / D\left(A_{\min }\right) \cong L_{A}^{\delta} / L_{A}^{\delta+\mu-0} .
$$

Proof. (B.3) is a consequence of elliptic regularity, while (B.4) follows from the description given in Proposition B.3 (c) and interpolation.

\section{ACKNOWLEDGMENT}

I would like to thank the referee for a number of valuable remarks. 


\section{REFERENCES}

1. E.A. Coddington and N. Levinson, Theory of ordinary differential equations, McGraw-Hill, New York, 1955. MR0069338(16:1022b)

2. S. Coriasco, E. Schrohe, and J. Seiler, Differential operators on conic manifolds: Maximal regularity and parabolic equations, Bull. Soc. Roy. Sc. Liege 70 (2001), 207-229. MR.1904055 (2003j:58039)

3. J.B. Gil, T. Krainer, and G.A. Mendoza, Geometry and spectra of closed extensions of elliptic cone operators, to appear in Canadian J. Math.

4. J.B. Gil and G.A. Mendoza, Adjoints of elliptic cone operators, Amer. J. Math. 125 (2003), 357-408. MR1963689 (2004h:58032)

5. I.C. Gohberg and E.I. Sigal, An operator generalization of the logarithmic residue theorem and the theorem of Rouché, Math. USSR Sbornik 13 (1971), 603-625. MR0313856 (47:2409)

6. P. Grisvard, Elliptic problems in nonsmooth domains, Monogr. Stud. Math., vol. 24, Pitman, Boston, 1985. MR0775683 (86m:35044)

7. M.V. Keldysh, On the eigenvalues and eigenfunctions of certain classes of non-selfadjoint linear operators, Dokl. Akad. Nauk SSSR 77 (1951), 11-14, In Russian.

8. V.A. Kondratiev, Boundary value problems for elliptic equations in domains with conical or angular points, Trudy Moskov. Mat. Obshch. 16 (1967), 209-292, In Russian. MR0226187 $(37: 1777)$

9. V.A. Kozlov and V.G. Maz'ya, Differential equations with operator coefficients with applications to boundary value problems for partial differential equations, Springer Monogr. Math., Springer, Berlin, 1999. MR1729870 (2001d:34090)

10. M. Lesch, Operators of Fuchs type, conical singularities, and asymptotic methods, TeubnerTexte Math., vol. 136, B. G. Teubner, Stuttgart, 1997. MR.1449639 (98d:58174)

11. J.-L. Lions and E. Magenes, Problèmes aux limites non homogènes et applications, Vol. 1, Dunod, Paris, 1968. MR0247243 (40:512)

12. X. Liu and I. Witt, Asymptotic expansions for bounded solutions to semilinear Fuchsian equation, Doc. Math. 9 (2004), 207-250. MR2117414 (2005m:35116)

13. R.B. Melrose, Transformation of boundary value problems, Acta Math. 147 (1981), 149-236. MR.0639039 (83f:58073)

14. S.A. Nazarov and B.A. Plamenevskij, Elliptic problems in domains with piecewise smooth boundaries, de Gruyter Exp. Math., vol. 13, de Gruyter, Berlin, 1994. MR1283387(95h:35001)

15. S. Rempel and B.-W. Schulze, Asymptotics for elliptic mixed boundary problems, Math. Research, vol. 50, Akademie-Verlag, Berlin, 1989. MR1002573 (90h:35083)

16. B.-W. Schulze, Pseudo-differential operators on manifolds with singularities, Stud. Math. Appl., vol. 24, North-Holland, 1991. MR.1142574 (93b:47109)

17. Appl. Math., J. Wiley, Chichester, 1998. MR1631763 (99m:35281)

18. I. Witt, Explicit algebras with the Leibniz-Mellin translation product, Math. Nachr. 280 (2007), 326-337. MR2292154

19. _ Asymptotic algebras, Microlocal Analysis and Asymptotic Analysis of PDE, RIMS Kokyuroku, vol. 1211, Kyoto University, Kyoto, 2001, pp. 21-33. MR.1874953

20. - On the factorization of meromorphic Mellin symbols, Advances in Partial Differential Equations (Parabolicity, Volterra Calculus, and Conical Singularities) (S. Albeverio, M. Demuth, E. Schrohe, and B.-W. Schulze, eds.), Oper. Theory Adv. Appl., vol. 138, Birkhäuser, Basel, 2002, pp. 279-306. MR.1966207 (2004c:35440)

21. _ Local asymptotic types, Manuscripta Math. 115 (2004), 1-17. MR 2092773 (2005g:47018)

Department of Mathematics, Imperial College London, 180 Queen's Gate, London SW7 2AZ, United Kingdom

Current address: Mathematical Institute, University of Göttingen, Bunsenstr. 3-5, D-37073 Göttingen, Germany

E-mail address: iwitt@uni-math.gwdg.de 